\title{
The effects of topological charge change in heavy ion collisions: "Event by event $\mathcal{P}$ and $\mathcal{C P}$ violation"
}

\author{
Dmitri E. Kharzeev, a Larry D. McLerran $\stackrel{a}{a}$ and \\ Harmen J. Warringa \\ a Department of Physics, Brookhaven National Laboratory, Upton NY 11973, USA \\ b RIKEN BNL Research Center, Brookhaven National Laboratory, Upton, NY \\ 11973, USA
}

\begin{abstract}
Quantum chromodynamics (QCD) contains field configurations which can be characterized by a topological invariant, the winding number $Q_{\mathrm{w}}$. Configurations with nonzero $Q_{\mathrm{w}}$ break the charge-parity $(\mathcal{C P})$ symmetry of QCD. We consider a novel mechanism by which these configurations can separate charge in the presence of a background magnetic field - the "Chiral Magnetic Effect". We argue that sufficiently large magnetic fields are created in heavy ion collisions so that the Chiral Magnetic Effect causes preferential emission of charged particles along the direction of angular momentum. Since separation of charge is $\mathcal{C P}$-odd, any observation of the Chiral Magnetic Effect could provide a clear demonstration of the topological nature of the QCD vacuum. We give an estimate of the effect and conclude that it might be observed experimentally.
\end{abstract}

\section{Introduction}

A remarkable property of gauge theories is the existence of topologically nontrivial configurations of gauge fields [1]. In Minkowski space-time, these configurations are associated with tunneling between different states which are related by topologically non-trivial gauge transformations [2,3]. These different states are characterized by the Chern-Simons topological charge [4]. Transitions between these different states which involve changes in the topological charge induce anomalous processes, that is, processes which are forbidden by the classical equations of motion of the theory, but when the theory is quantized, become allowed [5,6]. The amplitudes of such transitions vanish order by order in perturbation theory, and are associated with intrinsically nonperturbative phenomena involving many quanta. In electroweak theory, such 
transitions are associated with $B+L$ violation, where $B$ is baryon number and $L$ is lepton number. In QCD, they are tied to chirality non-conservation. Chirality conservation is an exact symmetry of the classical equations of motion of QCD and of perturbation theory in the zero quark mass limit. The zero quark mass limit should be a good approximation for high energy processes

It was originally thought that non-perturbative phenomena associated with the underlying topology were suppressed by factors such as $e^{-2 \pi / \alpha}$ where $\alpha$ is the interaction strength of the gauge theory. This is typical of results from computation of quantum tunneling between states with different topological charge. It was a surprise when Manton and Klinkhamer proposed that for processes at high temperature, that the exponential suppression might disappear [7,8]. This is because at high temperatures, there is sufficient energy to pass classically over the barrier which separates states of different topological charge. Kuzmin, Rubakov and Shaposhnikov argued that the rate would be sufficiently large so that these processes might be important for electroweak baryogenesis [9,10]. The rates for such processes were computed [11], and it was eventually understood that such rates were not exponentially suppressed because they involved the coherent interaction of many low-energy quanta in an initial state going to many quanta in a final state [12]. Such rates should be large therefore not only at finite temperature but also in out-of-equilibrium processes which involve many particles. It was also shown that the computations for electroweak baryon number violation have a counterpart in helicity non-conservation in QCD [13].

An interesting feature of the transitions changing topological charge is that they involve $\mathcal{P}$ - and $\mathcal{C P}$ - odd field configurations. Because of this, $\mathcal{P}$ - and $\mathcal{C} \mathcal{P}-$ invariance of strong interactions is no longer "natural". These symmetries can however be imposed dynamically by postulating the existence of axions [14,15,16]; see [17] for reviews. In any case, $\mathcal{P}$ - and $\mathcal{C} \mathcal{P}$-invariance of strong interactions has to be related to the vacuum structure. It is thus possible that an excited vacuum domain which may be produced in heavy ion collisions as proposed by Lee and Wick [18] can break parity and and $\mathcal{C P}$ spontaneously [19]. Some consequences of such a scenario had been studied by Morley and Schmidt [20], who proposed as a signature $\mathcal{C P}$-odd correlations between the spins of the protons produced in a heavy ion collision. Other realizations of an excited vacuum domain (including the ones with parity-odd fluctuations) have been considered in Refs [21].

It was proposed that in the vicinity of the deconfinement phase transition QCD vacuum can possess metastable domains leading to $\mathcal{P}$ and $\mathcal{C P}$ violation [22]. It was also suggested that this phenomenon would manifest itself in specific correlations of pion momenta [22,23]. (For related studies of metastable vacuum states, especially in supersymmetric theories, see [24,25,26]). The existence of $\mathcal{P}$-odd bubbles had been inferred from the analysis of an effective chiral theory incorporating axial anomaly [22]; such bubbles may also be 
viewed as the space-time domains occupied by gauge field configurations with non-trivial winding number.

The existence of metastable $\mathcal{P}-$ odd bubbles does not contradict the VafaWitten theorem [27] stating that $\mathcal{P}$ and $\mathcal{C P}$ cannot be broken in the true ground state of QCD for $\theta=0$. Moreover, this theorem does not apply to QCD matter at finite isospin density [28] and finite temperature [29], where Lorentz-non-invariant $\mathcal{P}$-odd operators are allowed to have non-zero expectation values. For matter at zero temperature and finite baryon density, Migdal [30] long time ago advocated the possibility of parity-breaking pion condensate phase (for a recent work on $\mathcal{P}$-violation in cold dense matter, see [31] and references therein). Degenerate vacuum states with opposite parity were found [32] in the superconducting phase of QCD. A parity broken phase also exists in lattice QCD with Wilson fermions [33], but this phenomenon has been recognized as a lattice artifact for the case of mass-degenerate quarks; spontaneous $\mathcal{P}$ and $\mathcal{C P}$ breaking similar to the Dashen's phenomenon 34] can however occur for non-physical values of quark masses [35. $\mathcal{P}$-even, but $\mathcal{C}$-odd metastable states have also been argued to exist in hot gauge theories [36]. The conditions for the applicability of Vafa-Witten theorem have been repeatedly re-examined in recent years [37].

Several dynamical scenarios for the decay of $\mathcal{P}$-odd bubbles have been considered [38, and numerical lattice calculations of the fluctuations of topological charge in classical Yang-Mills fields have been performed [3940]. A closely related topic is the role of topological effects in high-energy scattering and multi-particle production which has been studied in Refs [41,42,43,44,45]. The studies of $\mathcal{P}$ - and $\mathcal{C P}$-odd correlations of pion momenta [46,47] have shown that such measurements are in principle feasible but would require large event samples.

Some time ago, it was pointed out that the presence of non-zero angular momentum (or equivalently of magnetic field) in heavy ion collisions makes it possible for $\mathcal{P}$ - and $\mathcal{C P}$-odd domains to induce charge separation in the produced particles [48. This is because the $\mathcal{P}$ - and $\mathcal{C} \mathcal{P}$-breaking term carries net chirality and thus generates asymmetry between left- and right-handed fermions $\sim \bar{\psi}_{L} \psi_{R}-\bar{\psi}_{R} \psi_{L} \neq 0$. In the presence of a magnetic field a $\mathcal{P}-$ and $\mathcal{C P}$-odd domain can generate chirality not by flipping the spins of the quarks, but by inducing up-down asymmetry (as measured with respect to the symmetry axis of the angular momentum) in the production of quarks and anti-quarks [48]. The magnitude of the effect has been estimated, and found to be within experimental reach. A very useful experimental observable measuring the charge separation with respect to reaction plane (which is perpendicular to the angular momentum and magnetic field axis) has been proposed by Voloshin [49] shortly afterwards. The first result of a dedicated experimental study of the effect has been recently presented by the STAR Collaboration at RHIC [50].

In Ref. [51] it has been noticed that the effect of charge separation can also 
be understood in the following way. At finite $\theta$-angle (as present effectively in a $\mathcal{P}$ - and $\mathcal{C} \mathcal{P}$-odd vacuum domain), a magnetic field $\boldsymbol{B}$ induces through the axial anomaly a parallel electric field $\sim \theta \boldsymbol{E}$, and a corresponding electric dipole moment. One may consider as a theoretical illustration of this phenomenon the transformation, at finite $\theta$, of a magnetic monopole into a "dyon" with an electric charge $\sim-e \theta / 2 \pi$ demonstrated by Witten [52]. In an off-central heavy ion collision, a $\mathcal{P}$ - and $\mathcal{C} \mathcal{P}$-odd vacuum domain was shown [51] to induce an electric field perpendicular to the reaction plane of magnitude $E_{z} \sim$ $-(e \theta / 2 \pi) l$, where $l \sim b$ is the angular momentum in a collision at impact parameter $b$. The corresponding electric charge separation has been estimated and found amenable to experimental observation.

In this paper we discuss a novel mechanism for charge separation. The topological charge changing transitions provide the $\mathcal{P}$ - and $\mathcal{C P}$ - violation necessary for charge separation. The variance of the net topological charge change is proportional to the total number of topological charge changing transitions. Hence if sufficiently hot matter is created in heavy ion collisions so that topological charge transitions can take place, we expect on average in each event a finite amount of topological charge change.

Charge separation needs a symmetry axis along which the separation can take place. The only symmetry axis in a heavy ion collision is angular momentum which points in the direction perpendicular to the reaction plane. In central collisions there is no symmetry axis, so in that case charge separation should vanish.

If charge separation would manifest itself mainly into pions it should violate isospin symmetry (interchange of up and down quarks). This is because under an isospin transformation a $\pi^{+}$becomes a $\pi^{-}$meson, so in an isospin symmetric situation $\pi^{+}$should behave similar to $\pi^{-}$, hence in that case they can never separate. Isospin violation can occur because up and down quarks have a different mass, because our initial state could be isospin asymmetric, and because up and down quarks have different charge, or because of the presence of an electromagnetic field.

Since QCD contains configurations that break the $\mathcal{C P}$ symmetry, but not the $\mathcal{C}$ symmetry, the charge separation mechanism we are looking for should not violate $\mathcal{C}$. However, a particle with positive charge transforms to a particle with negative charge under charge conjugation. In order to maintain $\mathcal{C}$ invariance, some important ingredient of the mechanism that separates charge should transform under $\mathcal{C}$ as well. The only possibility lies in the initial state, since the incoming ions have a positive electric charge, they transform under $\mathcal{C}$. So our mechanism should somehow depend on the electric charges of the ions.

Summarizing, the charge separation mechanism should provide a symmetry axis, it should violate isospin and depend on the charges of the colliding ions. Therefore it is very natural that the (electromagnetic) magnetic field 
is crucial for charge separation. This magnetic field depends on the electric charges of the colliding nuclei, so that $\mathcal{C}$ invariance is maintained. The magnetic field provides a symmetry axis; midway between the two colliding nuclei, the magnetic field is parallel to the angular momentum vector. Moreover the magnetic field causes violation of isospin since up and down quarks will be polarized oppositely in a magnetic field.

We can understand the effect we will discuss in this article in a simple

example. Suppose we have a box with some net topological charge. This means that due to the anomaly, there is a net helicity for the quarks inside the box. If the box is at high enough temperature, then perturbative interactions which involve spin flip are negligible, since $T \gg m_{\text {quark }}$, so the net helicity is directly proportional to the net topological charge. Now imagine we apply an electrodynamic magnetic field. This will orient the spin of positively charged quarks (and anitquarks) along the direction of the magnetic field, due to the magnetic moment interaction. Likewise, the spins of negatively charged quarks (and anitquarks) will become oriented opposite to the direction of magnetic field. Now because there is net helicity, the orientation of spin is preferentially in the direction of momentum of the particles. Positively charged quarks and antiquarks move in the direction of the magnetic field, and negatively charged quarks and antiquarks move opposite to it. Therefore an electromagnetic current is set up in the direction of the magnetic field. We therefore call this the Chiral Magnetic Effect. The Chiral Magnetic Effect induces a vector current. For systems of a finite size, this current results in charge separation or an induced electric dipole moment.

The separation is as required $\mathcal{C}$ even, since under $\mathcal{C}$ both the magnetic field and the vector current change sign. Under $\mathcal{P}$, the current changes sign, but the magnetic field does not, so it is $\mathcal{P}$ odd, and $\mathcal{C P}$ odd.

This article is organized as follows. In Section 2 and 3 we review the axial anomaly and the QCD vacuum structure respectively. In Section 4 we discuss in detail the Chiral Magnetic Effect. Section 5 is devoted to the consequences of this effect in heavy ion collisions. In Section 6 we define some observables and Section 7 contains a discussion of our results. The derivation of the magnetic field is discussed in Appendix A. An alternative derivation of the Chiral Magnetic Effect is given in Appendix B.

\section{The Axial Anomaly}

In this section, we review some properties of topologically non-trivial gauge fields. We also review the relationship between topology and anomalous processes.

All gauge field configurations which have finite action can be characterized by a topological invariant, the winding number $Q_{\mathrm{w}}$. The winding number is 
an integer and given by

$$
Q_{\mathrm{w}}=\frac{g^{2}}{32 \pi^{2}} \int \mathrm{d}^{4} x F_{\mu \nu}^{a} \tilde{F}_{a}^{\mu \nu} \in \mathbb{Z} .
$$

Here $g$ denotes the QCD coupling constant with generators normalized as $\operatorname{tr} t_{a} t_{b}=\delta_{a b} / 2$. The gluonic field tensor and its dual are respectively $F_{\mu \nu}^{a}$ and $\tilde{F}_{\mu \nu}^{a}=\frac{1}{2} \epsilon_{\mu \nu}^{\rho \sigma} F_{\rho \sigma}^{a}$. Configurations with nonzero $Q_{\mathrm{w}}$ lead to non-conservation of axial currents even in the chiral limit. This can be seen from the axial Ward-identity which reads

$$
\partial^{\mu} j_{\mu}^{5}=2 \sum_{f} m_{f}\left\langle\bar{\psi}_{f} i \gamma_{5} \psi_{f}\right\rangle_{A}-\frac{N_{f} g^{2}}{16 \pi^{2}} F_{\mu \nu}^{a} \tilde{F}_{a}^{\mu \nu}
$$

here $N_{f}$ denotes the number of quark flavors, $\psi_{f}$ a quark field, and $m_{f}$ the mass of a quark. The axial current $j_{\mu}^{5}$ is given by

$$
j_{\mu}^{5}=\sum_{f}\left\langle\bar{\psi}_{f} \gamma_{\mu} \gamma_{5} \psi_{f}\right\rangle_{A}
$$

We write $\langle O\rangle_{A}$ to denote an average over fermionic fields only in the background of a particular gauge field configuration $A_{\mu}$. To obtain the full expectation value $\langle O\rangle$ one still has to perform the integration over the gluonic fields $A_{\mu}$. In this article we will only consider the chiral limit, i.e. $m_{f}=0$.

Assuming that initially at $t=-\infty$ we have an equal number of righthanded and left-handed fermions 1 , i.e. $N_{R}=N_{L}$, it follows from the axial Ward identity that at $t=\infty$

$$
\left(N_{L}-N_{R}\right)_{t=\infty}=2 N_{f} Q_{\mathrm{w}}
$$

This shows that configurations with positive $Q_{\mathrm{w}}$ convert right-handed fermions into left-handed ones.

Let us now write the number of right- or left-handed fermions of a particular flavor as $N_{R, L}^{f}$. So $N_{R, L}=\sum_{f} N_{R, L}^{f}$. Assuming that initially for each flavor separately $N_{R}^{f}=N_{L}^{f}$, one can show that $t=\infty$

$$
\left(N_{L}^{u}-N_{R}^{u}\right)_{t=\infty}=\left(N_{L}^{d}-N_{R}^{d}\right)_{t=\infty}
$$

where in this equation $u$ and $d$ can denote any quark flavor. This implies that in the chiral limit, the number of converted up and down quarks is equal.

1 With fermions we mean quarks and antiquarks. We write $N_{R}$ for the total number of right-handed fermions, which is the sum of the number of right-handed particles and right-handed antiparticles. Here right-handed massless fermions have spin and momentum parallel, while left-handed massless fermions have spin and momentum antiparallel. The volume integral over $\psi_{R}^{\dagger} \psi_{R}$ however, is equal to the total number of right-handed particles minus the total number of left-handed antiparticles. 


\section{The QCD Vacuum}

In the classical vacuum of QCD the gauge field has to be a pure gauge so that the energy density is minimal. In the temporal gauge $\left(A_{0}=0\right)$ this implies $A_{i}(\boldsymbol{x})=\frac{i}{g} U(\boldsymbol{x}) \partial_{i} U^{\dagger}(\boldsymbol{x})$, where $U(\boldsymbol{x})$ is an element of the gauge group $\mathrm{SU}(3)$. The different classical vacua can be characterized by a topological invariant, the winding number $n_{\mathrm{w}}$ which is an integer and given by

$$
n_{\mathrm{w}}=\frac{1}{24 \pi^{2}} \int \mathrm{d}^{3} x \epsilon^{i j k} \operatorname{tr}\left[\left(U^{\dagger} \partial_{i} U\right)\left(U^{\dagger} \partial_{j} U\right)\left(U^{\dagger} \partial_{k} U\right)\right]
$$

Now one can show that if a gauge field configuration goes to a pure gauge at infinity and has nonzero $Q_{\mathrm{w}}$ it induces a transition from one classical vacuum to another, more precisely

$$
Q_{\mathrm{w}}=n_{\mathrm{w}}(t=\infty)-n_{\mathrm{w}}(t=-\infty) .
$$

At zero temperature such transition requires tunneling through a potential barrier, which will suppress the transition rate exponentially. In this case the main contribution to the transition rate comes from fluctuations around instantons (which are minima of the classical Euclidean action). It was found by 't Hooft [2] (see also [53]) in QCD without fermions that the number of transitions $N_{\mathrm{t}}^{ \pm}$with $Q_{\mathrm{w}}= \pm 1$ per unit volume and time equals

$$
\frac{\mathrm{d} N_{\mathrm{t}}^{ \pm}}{\mathrm{d}^{3} x \mathrm{~d} t \mathrm{~d} \rho}=0.0015\left(\frac{2 \pi}{\alpha_{S}}\right)^{6} \exp \left(-\frac{2 \pi}{\alpha_{S}}\right) \frac{1}{\rho^{5}},
$$

here $\rho$ denotes the size of an instanton and $\alpha_{S}=g^{2}(\rho) /(4 \pi)$ the renormalized coupling constant.

At finite temperature the size of an instanton cannot be larger than $1 /(g T)$ because of Debye screening. Because large instantons cannot contribute to the transition rate at large $T$ anymore and $g(T)$ goes to zero due to asymptotic freedom, the transition rate due to instantons will go down if the temperature is raised. More specifically Pisarski and Yaffe [54] computed the transition rate in the absence of fermions and found

$$
\frac{\mathrm{d} N_{\mathrm{t}}^{ \pm}}{\mathrm{d}^{3} x \mathrm{~d} t \mathrm{~d} \rho}=\left.\exp \left(-2 \pi^{2} \rho^{2} T^{2}-18 A(\pi \rho T)\right) \frac{\mathrm{d} N_{\mathrm{t}}^{ \pm}}{\mathrm{d}^{3} x \mathrm{~d} t \mathrm{~d} \rho}\right|_{T=0}
$$

where $A(\lambda)$ is a function which behaves as $-\log (\lambda) / 6$ for $\lambda \rightarrow \infty$ and as $-\lambda^{2} / 36$ for $\lambda \rightarrow 0$ [55].

Hence for high temperatures the transition rate due to instantons becomes extremely small. This also happens in the electroweak theory where the coupling constant is always tiny. In this case transitions between different vacua lead to violation of baryon plus lepton $(B+L)$ number [2]. However, it was

realized that the electroweak theory contains static solutions which have finite 
energy and half integer winding number [8]. Such a solution with minimal energy is called a sphaleron (ready to fall). If the temperature is higher than the energy of the sphaleron it is likely that one can go over the barrier instead of tunneling. This increases the transition rate and hence the rate for baryon number violation enormously [9, 10, 11, 12,

It was realized that these sphaleron configurations also exist in QCD [13]. The typical energy of the sphalerons in QCD is $\Lambda_{\mathrm{QCD}} \approx 200 \mathrm{MeV}$. Hence also in QCD at high temperatures one can go over the barrier which leads to an enormous increase in the transition rate. According to Refs. [56]-[57] the rate for $\mathrm{SU}(2)$ Yang-Mills theory is

$$
\frac{\mathrm{d} N_{\mathrm{t}}^{ \pm}}{\mathrm{d}^{3} x \mathrm{~d} t} \sim 25.4 \alpha_{W}^{5} T^{4}
$$

The rate has not been directly measured for QCD, but if we assume $N_{c}$ scaling, we estimate the rate for QCD to be

$$
\frac{\mathrm{d} N_{\mathrm{t}}^{ \pm}}{\mathrm{d}^{3} x \mathrm{~d} t} \equiv \Gamma^{ \pm} \sim 192.8 \alpha_{S}^{5} T^{4}
$$

There is a subtlety in these considerations for massless quarks. For massless quarks, the overlap between a state with zero Chern-Simons charge, and one with one unit of charge vanishes,

$$
<n_{w}=1 \mid n_{w}=0>=0,
$$

since in the path integral representation for such a transition, the fermion determinant has a zero mode and vanishes. However, there is a non-zero contribution for

$$
<n_{w}=1|\bar{\psi} \psi| n_{w}=0>
$$

since in the path integral representation for this quantity, the zero from the determinant is canceled by a singularity from the quark propagator which represents the operator $\bar{\psi} \psi$. The overall expression is proportional to zero mode wavefunctions. This operator corresponds to a chirality flip, and as it should satisfies the anomaly relation between the change of helicity and the change of Chern-Simons charge.

The total rate of transition is of course the sum of the rates of the lowering and raising transitions,

$$
\frac{\mathrm{d} N_{\mathrm{t}}}{\mathrm{d}^{3} x \mathrm{~d} t}=\sum_{ \pm} \frac{\mathrm{d} N_{\mathrm{t}}^{ \pm}}{\mathrm{d}^{3} x \mathrm{~d} t}
$$

\section{The Chiral Magnetic Effect}

We will show in this section that gauge field configurations with nonzero $Q_{\mathrm{w}}$ can separate charge in the presence of a background (electromagnetic) magnetic field $B$. This we call the Chiral Magnetic Effect. We consider the 
most ideal situation in which all quark masses vanish. This should be a good approximation in the deconfined, chiral symmetry restored phase. First we consider the case with a very large magnetic field, which means for us that $e B$ is much larger than the momentum squared of all particles, i.e. $e B \gg p^{2}$. Then we will study the effect of a moderate field.

\subsection{Large magnetic field}

Initially we assume that we have a very small number of left and right-handed fermions. This is because our initial state has little fluctuation in the helicities of particles. This is a reasonable assumption for an initial state typical of a Color Glass Condensate, where the coupling is weak and the fluctuations should be generated by quantum mechanical tunneling, and therefore suppressed as $\exp \left(-2 \pi / \alpha_{S}\right)$ [58,59,42. At later time, sphaleron-like transitions should be possible since the system becomes an ensemble of classical configurations with energy typically greater than the barrier [60].

Since the magnetic field is so large, all particles will be found in the lowest Landau level. Hence their spin is aligned along the magnetic field and they can also only move along the magnetic field. Quarks with opposite charges have their spins aligned in different directions. If $B$ points in the $z$-direction positively charged right-handed fermions and negatively charged left-handed fermions will move in the positive $z$-direction (upwards). At the same time positively charged left-handed fermions and negatively charged right-handed fermions will move downwards. We illustrated this situation in Fig. 1.

The fermions will then interact with a gauge field configuration, so that some of them will change their helicity. After the interaction with the gauge field configuration has taken place we find that (since we assumed the chiral limit)

$$
\left(N_{L}^{f}-N_{R}^{f}\right)=2 Q_{\mathrm{w}},
$$

so that if $Q_{\mathrm{w}}$ is nonzero we obtain a difference between the number of left and right-handed fermions that is the same for each flavor. The fermions can only change helicity by reversing their momenta, since spin flip is energetically suppressed in a large magnetic field (we assumed $e B \gg p^{2}$ ). So after the interaction has taken place all positively charged right-handed fermions and negatively charged left-handed fermions will still move upwards. At the same time positively charged left-handed quarks and negatively charged righthanded quarks will still move downwards. However, now there is a difference between the number of right-handed and left-handed fermions. As a result an electromagnetic current is generated along the direction of $B$. If we assume that initially all fermions are within a finite volume, this current will induce a charge difference $Q e$ between opposite sides a plane perpendicular to the magnetic field. Here $e$ denotes the elementary charge and

$$
Q=2 Q_{\mathrm{w}} \sum_{f}\left|q_{f}\right|
$$




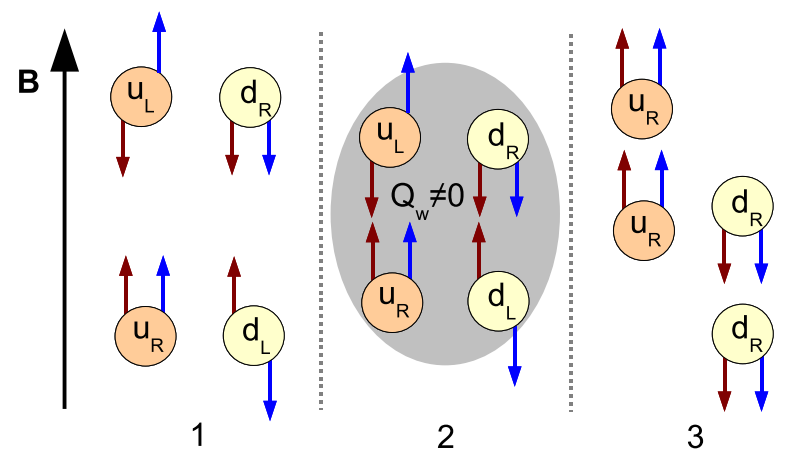

Fig. 1. Illustration of the Chiral Magnetic Effect in a very large homogeneous magnetic field. The red arrows denote the direction of momentum, the blue arrows - the spin of the quarks. (1) Due to the very large magnetic field the up and down quarks are all in the lowest Landau level and can only move along the direction of the magnetic field. Initially there are as many left-handed as right-handed quarks. (2) The quarks interact with a gauge configuration with nonzero $Q_{\mathrm{w}}$. Assuming $Q_{\mathrm{w}}=-1$, this will convert a left-handed up/down quark into a right-handed up/down quark by reversing the direction of momentum. (3) The right-handed up quarks will move upwards, the right-handed down quarks will move downwards. A charge difference of $Q=2 e$ will be created between two sides of a plane perpendicular to the magnetic field.

where $q_{f}$ is the charge in units of $e$ of a quark with flavor $f$. For $N_{f}=2$ and $N_{f}=3$ the relation above becomes $Q=2 Q_{w}$ and $Q=\frac{8}{3} Q_{w}$ respectively. An alternative derivation of this result is presented in Appendix B.

Since Eq. (16) was obtained in the most ideal case, i.e. chiral limit and an extremely large magnetic field, Eq. (16) has to be an upper limit. Therefore there is a maximum amount of charge that can be separated by a particular gauge field configuration in a homogeneous background magnetic field; in other words

$$
|Q| \leq 2\left|Q_{\mathrm{w}}\right| \sum_{f}\left|q_{f}\right|
$$

In our derivation we ignored the back-reaction due to the electric field created by the separating charges. We believe that this back-reaction can only give rise to a small suppression of the effect. This is because the size of the electric field will be much smaller than $e B$ since there are very few particles involved. Moreover, in the physical case we are interested in, a heavy ion collision, the color forces will surely dominate.

Since the separation is independent of color, the mechanism will not create a net color charge difference. Therefore we can safely ignore a gluonic backreaction.

\subsection{Moderate Magnetic Field}

Now that we considered the ideal situation with an extremely large magnetic field, let us estimate the amount of charge separated in a moderate homoge- 
neous magnetic field. There are now three important scales in the problem, $e B$, the temperature $T$, and the size $\rho$ of the configuration with nonzero $Q_{\mathrm{w}}$.

In a moderate magnetic field not all spins will be aligned along $B$. Let us introduce the function $\beta_{f}(\omega)$ which denotes the degree of polarization along $B$ of quarks with flavor $f$ and energy smaller than $\omega$. Since a magnetic field does not distinguish between left and right-handed particles, $\beta_{f}(\omega)$ will be the same for left and right-handed particles in thermodynamic equilibrium. Moreover, ignoring chemical potential, $\beta_{f}(\omega)$ will be equal for particles and antiparticles.

In a homogeneous magnetic field it is possible to calculate $\beta_{f}(\omega)$. The energy dispersion relation of a massless fermion is then given by

$$
\omega_{p}^{2}=p_{3}^{2}+2\left|q_{f} e B\right| n
$$

where $n=0,1,2, \ldots$ denotes the Landau level. The $n=0$ level contains only one spin direction, all other levels contain both spin directions and are hence double degenerate.

The number density of quarks in a magnetic field with energy smaller than $\omega$ reads

$$
N_{\uparrow}+N_{\downarrow}=\frac{\left|q_{f} e B\right|}{2 \pi^{2}} \sum_{n=0}^{\infty} \int_{0}^{\infty} \mathrm{d} p_{3} \alpha_{n} \theta\left(\omega^{2}-\omega_{p}^{2}\right) n\left(\omega_{p}\right),
$$

where $\alpha_{n}=2-\delta_{n, 0}$ is the degeneracy factor and $n\left(\omega_{p}\right)=\left[\exp \left(\omega_{p} / T\right)+1\right]^{-1}$ is the Fermi-Dirac distribution function. The difference between the number density of fermions with spin parallel and antiparallel to the magnetic field yields

$$
\left|N_{\uparrow}-N_{\downarrow}\right|=\frac{\left|q_{f} e B\right|}{2 \pi^{2}} \int_{0}^{\infty} \mathrm{d} p_{3} \theta\left(\omega^{2}-p_{3}^{2}\right) n\left(p_{3}\right) .
$$

Hence the degree of polarization of quarks/antiquarks with energy smaller than $\omega$ is given by

$$
\beta_{f}(\omega)=\frac{\left|N_{\uparrow}-N_{\downarrow}\right|}{N_{\uparrow}+N_{\downarrow}} .
$$

Let us try to find an approximation for $\beta_{f}(\omega)$. For $2\left|q_{f} e B\right|>\omega^{2}, \beta_{f}(\omega)=1$ since only the lowest Landau level contributes. For $2\left|q_{f} e B\right|=0, \beta_{f}(\omega)=0$. Therefore

$$
\beta_{f}(\omega) \approx \gamma\left(\frac{2\left|q_{f} e B\right|}{\omega^{2}}\right)
$$

with

$$
\gamma(x)=\left\{\begin{array}{l}
x \text { for } x \leq 1 \\
1 \text { for } x \geq 1
\end{array} .\right.
$$

We have computed Eq. (21) numerically and found that Eq. (22) is a reasonable approximation.

At first hand, it might seem surprising that the temperature drops out the estimate for the degree of polarization. The reason for this is that we 


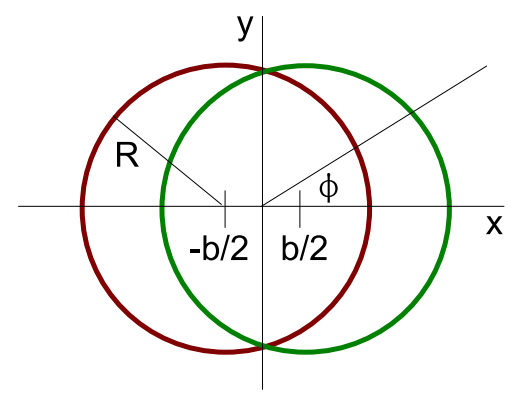

Fig. 2. Crossectional view of a non-central heavy ion collision along the beam-axis ( $z$-axis). The two nuclei have radii $R$, travel in opposite directions and collide with impact parameter $b$. The plane $y=0$ is called the reaction plane. The angle $\phi$ is an azimuthal angle with respect to the reaction plane. The region in which the two nuclei overlap contains the participants, the regions in which they do not contain the spectators.

only considered the degree of polarization of a subset of all modes, namely the ones with energy smaller than $\omega$. If one would instead be interested in the polarization of the bulk of the particles, one should take $\omega \approx T$, by which the temperature dependence naturally is recovered.

Quarks with momenta much larger than the inverse size $1 / \rho$ of the configuration with nonzero winding number will not be affected by these configurations and hence do not change helicity. Therefore the quarks that change helicity will have momenta and hence energy smaller than $\sim 1 / \rho$. Assuming that all quarks with energy smaller than $1 / \rho$ are equally likely to change helicity we find the following estimate for the expectation value of the amount of charge separated

$$
Q \approx 2 Q_{\mathrm{w}} \sum_{f}\left|q_{f}\right| \gamma\left(2\left|q_{f} \Phi\right|\right) .
$$

where $\Phi=e B \rho^{2}$ is essentially the flux through a configuration with nonzero $Q_{\mathrm{w}}$. For small fields $\left(2\left|q_{f} e B\right|<\rho^{2}\right)$ this reduces to

$$
Q \approx 4 \Phi Q_{\mathrm{w}} \sum_{f} q_{f}^{2}
$$

\section{Chiral Magnetic Effect in Heavy Ion Collisions}

Now that we know how much charge is separated by a single configuration with winding number $Q_{\mathrm{w}}$, let us estimate the amount of charge separated in a heavy ion collision. We will assume that hot deconfined chirally restored matter is created, so our results of the previous section will be applicable.

As we have shown in the previous section, if $e B \sim 1 / \rho^{2}$ there is a good chance of getting charge separation. In a hot plasma, the typical size of a configuration with nonzero $Q_{\mathrm{w}}$ is of the order of the magnetic screening length, $\alpha_{S} T$. The typical required magnetic fields are therefore of order $\alpha_{S}^{2} T^{2}$, 
which we estimate to be of order $10^{2}-10^{3} \mathrm{MeV}^{2}$, for the initial conditions typical at RHIC. In Appendix A, we show that such enormous magnetic fields can indeed be created in off-central heavy ion collisions. In the plasma, the magnetic field is to a good degree homogeneous and pointing in a direction perpendicular to the reaction plane ( $y=0$ in Fig. (2) $)$ in the region of overlap of the colliding nuclei for the impact parameters of interest.

We will now define $N_{\mathrm{a}}^{ \pm}$and $N_{\mathrm{b}}^{ \pm}$to be the total positive/negative charge in units of $e$ above $(a)$ and below $(b)$ the reaction plane respectively. We have that $N^{ \pm}=N_{\mathrm{a}}^{ \pm}+N_{\mathrm{b}}^{ \pm}$, where $N^{ \pm}$is the total positive/negative charge produced in a certain event. We define $\Delta_{ \pm}$to be the difference between in charge on each side of the reaction plane $\Delta_{ \pm}=N_{\mathrm{a}}^{ \pm}-N_{\mathrm{b}}^{ \pm}$.

One generates an electric current along the magnetic field each time a transition from one vacuum to another is made. Let us now imagine such transition happening in the center of the hot matter. Locally a charge difference will be created due to the current. But since the quarks creating this current will still have many interactions before they leave the hot matter, presumably transitions in the center due to the final-state randomization of quark momenta will not give rise to any observable charge separation.

Now let us look to a transition near the surface of the hot matter. Suppose that the situation is such that the charge separation mechanism causes more positively charged quarks to move outwards and more negatively charged quarks the move inwards (the opposite situation is of course also possible with an equal probability). The positively charged quarks moving out of the surface will hadronize and can essentially freely propagate to the detector. On the other hand the negatively charged quarks will move inwards and still encounter many interactions. As a result the azimuthal angles of the positively and negatively charged quarks will become uncorrelated. This is very similar to the observed jet quenching in heavy ion collisions. Only for very large impact parameters, when the size of the interacting region is about same as the scattering length, we expect correlations between positively and negatively charged particles to exist.

Each time a transition is made both $\Delta_{+}$and $\Delta_{-}$will change. The expectation value of the change is either positive or negative with equal probability and given by

$$
\pm \sum_{f}\left|q_{f}\right| \gamma\left(2\left|q_{f} \Phi\right|\right) \xi_{ \pm}\left(x_{\perp}\right)
$$

Here we used that the most probable transitions are those which connect two neighboring vacua, so $Q_{\mathrm{w}}= \pm 1$. The transitions which simultaneously induce changes with $Q_{\mathrm{w}}>1$ are suppressed, therefore we have neglected them. We compute those transitions which are independent of one another, but taken over the time and volume of the system can induce multiple changes in the Chern-Simons charge. The functions $\xi_{ \pm}\left(x_{\perp}\right)$ are screening suppression functions in order to incorporate the effect that transitions near the surface 
are much more likely to contribute to $\Delta_{ \pm}$. We will take

$$
\xi_{ \pm}\left(x_{\perp}\right)=\exp \left(-\left|y_{ \pm}(x)-y\right| / \lambda\right)
$$

where $\lambda$ is the screening length. The functions $y_{+}(x)$ and $y_{-}(x)$ denote the upper and lower $y$ coordinate of the overlap region. Hence $\left|y_{ \pm}(x)-y\right|$ is the distance from a point $y$ to the upper/lower part of the surface. It follows from Fig. (2) that

$$
y_{+}(x)=-y_{-}(x)=\left\{\begin{array}{lc}
\sqrt{R^{2}-(x-b / 2)^{2}} & -R+b / 2 \leq x \leq 0 \\
\sqrt{R^{2}-(x+b / 2)^{2}} & 0 \leq x \leq R-b / 2
\end{array},\right.
$$

here $R$ denotes the radius of the nuclei and $b$ is the impact parameter. Now one can easily infer from Eq. (26) that the screening works. It is equally likely to increase $\Delta_{ \pm}$as to decrease it. However if for example $y$ is near $y_{+}$ the expectation value for increasing $\Delta_{ \pm}$is much larger then the expectation value for decreasing it. Let us mention here that Eq. (26) is strictly speaking only valid for a constant homogeneous magnetic field. In the overlap region the magnetic field is to a good degree homogeneous around zero space-time rapditiy especially for large impact parameters. It has however a huge time dependence. So in reality there could be effects which are due to the rapid time variation of the field that we will ignore in this paper.

Now we define $N_{\mathrm{t}}^{ \pm}$to be the total number of raising/lowering transitions. It of course holds that $N_{\mathrm{t}}=N_{\mathrm{t}}^{+}+N_{\mathrm{t}}^{-}$. Furthermore we introduce $\Delta_{t}$ which is the difference between the number of raising and lowering transitions, i.e. $\quad \Delta_{\mathrm{t}}=N_{\mathrm{t}}^{+}-N_{\mathrm{t}}^{-}$. We can assume that all transitions happen independently from each other. Then the dynamics for $\Delta_{t}$ is exactly governed by a one-dimensional random walk. At each transition one has equal probability to go up or down. Hence the expectation value of $\Delta_{t}$ will vanish. But since there are $N_{\mathrm{t}}$ transitions its variation is equal to $\sqrt{N_{\mathrm{t}}}$, so that

$$
\left\langle\Delta_{\mathrm{t}}^{2}\right\rangle=\int_{t_{i}}^{t_{f}} \mathrm{~d} t \int_{V} \mathrm{~d}^{3} x \int \mathrm{d} \rho \frac{\mathrm{d} N_{\mathrm{t}}}{\mathrm{d}^{3} x \mathrm{~d} t \mathrm{~d} \rho}
$$

where $V$ denotes the volume in which the transitions take place.

Now we can compute the variation of $\Delta^{ \pm}$. Since we assume that all transitions are independent from each other the total variation is the sum of the variation of all contributions. Hence it follows that

$$
\begin{aligned}
\left\langle\Delta_{ \pm}^{2}\right\rangle=\frac{1}{2} \int_{t_{i}}^{t_{f}} \mathrm{~d} t \int_{V} \mathrm{~d}^{3} x \int \mathrm{d} \rho \frac{\mathrm{d} N_{\mathrm{t}}}{\mathrm{d}^{3} x \mathrm{~d} t \mathrm{~d} \rho}\left[\xi_{-}\left(x_{\perp}\right)^{2}+\xi_{+}\left(x_{\perp}\right)^{2}\right] \times & \\
& {\left[\sum_{f}\left|q_{f}\right| \gamma\left(2\left|q_{f} e B\right| \rho^{2}\right)\right]^{2} }
\end{aligned}
$$


Likewise we can compute $\left\langle\Delta_{+} \Delta_{-}\right\rangle$. We find

$$
\begin{aligned}
\left\langle\Delta_{+} \Delta_{-}\right\rangle=-\int_{t_{i}}^{t_{f}} \mathrm{~d} t \int_{V} \mathrm{~d}^{3} x \int \mathrm{d} \rho \frac{\mathrm{d} N_{\mathrm{t}}}{\mathrm{d}^{3} x \mathrm{~d} t \mathrm{~d} \rho} \xi_{-}\left(x_{\perp}\right) \xi_{+}\left(x_{\perp}\right) \times & \\
& {\left[\sum_{f}\left|q_{f}\right| \gamma\left(2\left|q_{f} e B\right| \rho^{2}\right)\right]^{2} }
\end{aligned}
$$

Let us remark here that if screening is absent we have $\left\langle\Delta_{ \pm}^{2}\right\rangle=-\left\langle\Delta_{+} \Delta_{-}\right\rangle$since then $\xi_{ \pm}=1$. In the presence of screening however, $\xi_{+} \xi_{-}<\frac{1}{2}\left(\xi_{+}^{2}+\xi_{-}^{2}\right)$ so that $\left|\left\langle\Delta_{+} \Delta_{-}\right\rangle\right|<\left\langle\Delta_{ \pm}^{2}\right\rangle$.

Using the fact that $\rho \sim\left(\Gamma^{ \pm} / \alpha_{S}\right)^{-1 / 4} \sim 1 /\left(\alpha_{S} T\right)$, and the expression for the sphaleron transition rate Eq. (11) we can rewrite the last two formula's for small magnetic fields $\left(2\left|q_{f} e B\right|<1 / \rho^{2}\right)$ as

$$
\begin{aligned}
& \frac{\mathrm{d}\left\langle\Delta_{ \pm}^{2}\right\rangle}{\mathrm{d} \eta}=2 \kappa \alpha_{S}\left[\sum_{f} q_{f}^{2}\right]^{2} \int_{V_{\perp}} \mathrm{d}^{2} x_{\perp}\left[\xi_{+}^{2}\left(x_{\perp}\right)+\xi_{-}^{2}\left(x_{\perp}\right)\right] \int_{\tau_{i}}^{\tau_{f}} \mathrm{~d} \tau \tau\left[e B\left(\tau, \eta, x_{\perp}\right)\right]^{2}, \\
& \frac{\mathrm{d}\left\langle\Delta_{+} \Delta_{-}\right\rangle}{\mathrm{d} \eta}=-4 \kappa \alpha_{S}\left[\sum_{f} q_{f}^{2}\right]^{2} \int_{V_{\perp}} \mathrm{d}^{2} x_{\perp} \xi_{+}\left(x_{\perp}\right) \xi_{-}\left(x_{\perp}\right) \int_{\tau_{i}}^{\tau_{f}} \mathrm{~d} \tau \tau\left[e B\left(\tau, \eta, x_{\perp}\right)\right]^{2},
\end{aligned}
$$

where the proper time $\tau=\left(t^{2}-z^{2}\right)^{1 / 2}$ and the space-time rapidity $\eta=$ $\frac{1}{2} \log [(t+z) /(t-z)]$. The volume integral is over the overlap region $V_{\perp}$ in the transverse plane. The time integral is from the initial time $\tau_{i}$ to a final time $\tau_{f}$. We have assumed here that the magnetic field does not change the sphaleron transition rate dramatically. We have also inserted a constant $\kappa$ which should be of order one, but with large uncertainties, since in our estimate of the scale size there is an uncertainty of order one which is taken to the fourth power. We assumed that the scale size factor in the sphaleron roughly canceled the factor of $385 \alpha_{S}^{4}$ in the numerator, since the rate per unit volume should be of the order of the inverse size of the typical field configuration to the fourth power.

At this point, we could take our exact expression for the magnetic field given in Appendix A and compute the rate. This we will do in a later paper for a variety of initial conditions. For now however we will use the approximate formulae for the magnetic field at the origin due to spectators and participants. The approximations are given in Eqs. (A.12) and (A.17). and valid for $R / \sinh \left(Y_{0}\right) \lesssim \tau \lesssim R$. Adding the contributions of the spectators and participants we find

$$
e B \approx Z \alpha_{E M}\left[c \exp \left(-Y_{0} / 2\right) \frac{1}{R^{1 / 2} \tau^{3 / 2}} f(b / R)+4 \exp \left(-2 Y_{0}\right) \frac{b}{\tau^{3}}\right]
$$

Here $Z$ denotes the charge of the nucleus, $R$ its radius and $Y_{0}$ the beam 
rapidity. The function $f(b / R)$ given in Eq. (A.18) and displayed in Fig. (A.2) describes the impact parameter dependence of the participant contribution to the magnetic field to first approximation. The constant $c=0.59907 \ldots$ and $\alpha_{E M} \approx 1 / 137$ denotes the fine structure constant. Especially for large impact parameters the magnetic field at the origin is very homogeneous in the transverse plane within the overlap region, so that Eq. (34) is a good approximation to the field at the surface of the interacting matter. For smaller impact parameters the magnetic field at the surface is somewhat smaller, so that we might overestimate the magnetic field at the surface using Eq. (34) for small impact parameters.

Let us assume that the screening length $\lambda$ is constant in this short time. Then the integral over the transverse plane can be done since we used that the magnetic field is homogeneous to a certain degree. We define

$$
\begin{aligned}
& g(b / R, \lambda / R) \equiv \frac{1}{2 R^{2}} \int_{V_{\perp}} \mathrm{d}^{2} x_{\perp}\left[\xi_{+}^{2}\left(x_{\perp}\right)+\xi_{-}^{2}\left(x_{\perp}\right)\right], \\
& h(b / R, \lambda / R) \equiv \frac{1}{R^{2}} \int_{V_{\perp}} \mathrm{d}^{2} x_{\perp} \xi_{+}\left(x_{\perp}\right) \xi_{-}\left(x_{\perp}\right) .
\end{aligned}
$$

In the limit of $\lambda \rightarrow \infty$ these functions become equal to

$$
\frac{1}{R^{2}} \int_{V_{\perp}} \mathrm{d}^{2} x_{\perp}=2 \arccos \left(\frac{b}{2 R}\right)-\frac{b}{R} \sqrt{1-\frac{b^{2}}{4 R^{2}}} .
$$

We can now perform the integration over proper time. In the integration over proper time, the magnetic field is sharply falling. We can therefore to good approximation take $\tau_{f}=\infty$ and find

$$
\begin{array}{r}
\frac{\mathrm{d}\left\langle\Delta_{ \pm}^{2}\right\rangle}{\mathrm{d} \eta}=4 Z^{2} \alpha_{E M}^{2} \kappa \alpha_{S}\left[\sum_{f} q_{f}^{2}\right]^{2} g(b / R, \lambda / R)\left[c^{2} \exp \left(-Y_{0}\right) f^{2}(b / R) \frac{R}{\tau_{i}}\right. \\
\left.+\frac{16}{5} c \exp \left(-5 / 2 Y_{0}\right) f(b / R) \frac{b R^{3 / 2}}{\tau_{i}^{5 / 2}}+4 \exp \left(-4 Y_{0}\right) \frac{b^{2} R^{2}}{\tau_{i}^{4}}\right] .
\end{array}
$$

We now must motivate a choice of initial time in the above equation. If the time at which the topological charge changing processes is less than the Lorentz contracted size of the system, then the magnetic field is at its maximum value, and our approximation of treating the spectator nucleons as being on an infinitesimally thin sheet breaks down. At the time when this approximation breaks down, the field should have achieved its maximum value. In this case, we should be able to approximate the initial time as

$$
\tau_{i}=\zeta R e^{-Y_{0}}
$$

where $\zeta \sim 2$. On the other hand, if the initial time for the topological charge 
changing processes is larger than this, we should be able to approximate

$$
\tau_{i} \sim 1 / Q_{\text {sat }}
$$

where $Q_{\text {sat }}$ is the saturation momentum. The saturation momentum sets the time scale for the evolution of classical fields produced in the collisions. The choice between these two times scales is the maximum of the two possible values.

For gold nuclei at $100 \mathrm{GeV}$ per nucleon, both these choices are roughly the same and of the order of $0.1-0.2 \mathrm{fm} / \mathrm{c}$. For copper at the same energy, the choice would be the inverse saturation momentum since a copper nucleus is much smaller than gold. For gold at lower energy, we would best choose the Lorentz contracted size scale, since the finite size of the nucleus becomes important.

If we use the Lorentz contracted size scale, appropriate for gold, then

$$
\begin{aligned}
\frac{\mathrm{d}\left\langle\Delta_{ \pm}^{2}\right\rangle}{\mathrm{d} \eta}=4 Z^{2} \alpha_{E M}^{2} \kappa \alpha_{S}\left[\sum_{f} q_{f}^{2}\right]^{2} g(b / R, \lambda / R)\left[c^{2} f^{2}(b / R) \frac{1}{\zeta}\right. & \\
& \left.+\frac{16}{5} c f(b / R) \frac{b}{R} \frac{1}{\zeta^{5 / 2}}+4 \frac{b^{2}}{R^{2}} \frac{1}{\zeta^{4}}\right]
\end{aligned}
$$

Similarly we find

$$
\begin{array}{rl}
\frac{\mathrm{d}\left\langle\Delta_{+} \Delta_{-}\right\rangle}{\mathrm{d} \eta}=-4 Z^{2} \alpha_{E M}^{2} \kappa \alpha_{S}\left[\sum_{f} q_{f}^{2}\right]^{2} & h(b / R, \lambda / R)\left[c^{2} f^{2}(b / R) \frac{1}{\zeta}\right. \\
+ & \left.\frac{16}{5} c f(b / R) \frac{b}{R} \frac{1}{\zeta^{5 / 2}}+4 \frac{b^{2}}{R^{2}} \frac{1}{\zeta^{4}}\right] .
\end{array}
$$

We expect that taking $\zeta=2$ is reasonable. For two flavors we get $\left(\sum_{f} q_{f}^{2}\right)^{2}=$ $25 / 81$. Then we find for collisions at large impact parameters when screening is unimportant

$$
\frac{\mathrm{d}\left\langle\Delta_{ \pm}^{2}\right\rangle}{\mathrm{d} \eta} \sim \frac{100}{81} Z^{2} \alpha_{E M}^{2} \alpha_{S} \kappa\left[2 \arccos \left(\frac{b}{2 R}\right)-\frac{b}{R} \sqrt{1-b^{2} / 4 R^{2}}\right] \frac{b^{2}}{4 R^{2}} .
$$

Now let us take $b / R=1.6$ which roughly corresponds to $50-60 \%$ centrality. For gold-gold collisions $(Z=79$ and $E / A=100 \mathrm{GeV})$ we then find taking $\alpha_{s}=1$ and $\kappa=1$

$$
\frac{\mathrm{d}\left\langle\Delta_{ \pm}^{2}\right\rangle}{\mathrm{d} \eta} \sim 0.1
$$

Let us stress again that this is a very rough estimate. For example taking the initial time twice as large will decrease our estimate by a factor 16 . We could easily increase our estimate by an order of magnitude by taking $\kappa=10$.

In the next section we will connect $\left\langle\Delta_{ \pm}^{2}\right\rangle$ and $\left\langle\Delta_{+} \Delta_{-}\right\rangle$to some observables. Then we will discuss in detail the implications of Eqs. (40) and (41). 


\section{Observables}

Due to the finite multiplicity of produced particles, one cannot observe charge separation in an individual event. The statistical fluctuations $\sim \sqrt{N}$ will be much larger than the expected charge asymmetry. By taking an average over many events $\Delta_{ \pm}$will of course vanish, since configurations with positive and negative $Q_{\mathrm{w}}$ are very likely to be produced with equal probability. However can define correlators which are sensitive to the variation $\left\langle\Delta_{ \pm}^{2}\right\rangle$. In this section we are going to connect these correlators to $\left\langle\Delta_{ \pm}^{2}\right\rangle$.

A set of very useful correlators for the study of charge separation was proposed by Voloshin [49]. For each event one defines

$$
f\left(\phi_{a}, \phi_{b}\right)=\frac{1}{N_{a} N_{b}} \sum_{i=0}^{N_{a}} \sum_{j=0}^{N_{b}} \cos \left(\phi_{a i}+\phi_{b j}\right),
$$

where $a, b= \pm$ denotes the charge, $N_{ \pm}$is the total number of positively or negatively charged particles, and $\phi_{a i}$ denotes the azimuthal angle of an individual charged particle with respect to the reaction plane.

Then in order to remove the multiplicity fluctuations one averages the correlators over $N_{\mathrm{e}}$ similar events. This averaging is called event mixing. In this way one can define the averaged correlators $a_{--}, a_{++}$and $a_{+-}$[49], where

$$
a_{a b}=-\frac{1}{N_{\mathrm{e}}} \sum_{n=1}^{N_{\mathrm{e}}} f\left(\phi_{a}, \phi_{b}\right)
$$

We feel that it is also useful to define correlators which are not divided by square of the total multiplicity of charged particles. Here we introduce $b_{--}$, $b_{++}$and $b_{+-}$, where

$$
b_{a b}=-\frac{1}{N_{\mathrm{e}}} \sum_{n=1}^{N_{\mathrm{e}}} g\left(\phi_{a}, \phi_{b}\right)
$$

with

$$
g\left(\phi_{a}, \phi_{b}\right)=\sum_{i=0}^{N_{a}} \sum_{j=0}^{N_{b}} \cos \left(\phi_{a i}+\phi_{b j}\right) .
$$

The correlators can also be written as

$$
g\left(\phi_{a}, \phi_{b}\right)=X_{a} X_{b}-Y_{a} Y_{b},
$$

where

$$
X_{ \pm} \equiv \sum_{i=1}^{N_{ \pm}} \cos \left(\phi_{i}^{ \pm}\right), \quad Y_{ \pm} \equiv \sum_{i=1}^{N_{ \pm}} \sin \left(\phi_{i}^{ \pm}\right) .
$$

An equivalent representation of the last two equations reads

$$
X_{ \pm}=\int_{0}^{2 \pi} \mathrm{d} \phi \cos (\phi) \frac{\mathrm{d} N_{ \pm}}{\mathrm{d} \phi}, \quad Y_{ \pm}=\int_{0}^{2 \pi} \mathrm{d} \phi \sin (\phi) \frac{\mathrm{d} N_{ \pm}}{\mathrm{d} \phi}
$$


where $\mathrm{d} N_{ \pm}$denotes the number of charged particles in the azimuthal angle $\mathrm{d} \phi$. Now one can see that $X_{ \pm}$essentially measures directed flow. By taking a symmetric interval in rapidity this quantity vanishes. On the other hand $Y_{ \pm}$measures the difference between charges on opposite sides of the reaction plane. Let us emphasize that if a measurement shows that $a_{++} / b_{++}$is positive there really is on average more charge on one particular side of the reaction plane. Possible contamination by directed flow will always give a negative contribution to $a_{++} / b_{++}$. The correlator $a_{+-} / b_{+-}$measures correlations between positively and negatively charged particles and the reaction plane. If such correlation exists and positively charged particles are emitted oppositely from negatively charged particles $a_{+_{-}} / b_{+_{-}}$is necessarily negative.

In order to relate the correlators to $\Delta_{ \pm}$we have to make an assumption of the distribution of the charges in the azimuthal angle. If all charged particles affected by the Chiral Magnetic Effect would be really emitted perpendicular to the reaction plane in which case $\phi=\pi / 2$ or $\phi=3 \pi / 2$ we would have

$$
Y_{ \pm}=\Delta_{ \pm}
$$

As a result

$$
a_{++}=a_{--}=\frac{1}{N_{+}^{2}}\left\langle\Delta_{ \pm}^{2}\right\rangle, \quad a_{+-}=\frac{1}{N_{+} N_{-}}\left\langle\Delta_{+} \Delta_{-}\right\rangle
$$

and

$$
b_{++}=b_{--}=\left\langle\Delta_{ \pm}^{2}\right\rangle, \quad b_{+-}=\left\langle\Delta_{+} \Delta_{-}\right\rangle .
$$

where $N_{ \pm}$denotes the total number of charged particles in the corresponding $\eta$ interval.

However, it might be more realistic that the particles will spread along the direction of the average magnetic field. If for example

$$
\frac{\mathrm{d} N_{ \pm}}{\mathrm{d} \phi}=\frac{1}{2 \pi} N_{ \pm}+\frac{1}{4} \Delta_{ \pm} \sin (\phi)
$$

we would find

$$
Y_{ \pm}=\frac{\pi}{4} \Delta_{ \pm}
$$

In that case

$$
a_{++}=a_{--}=\frac{1}{N_{+}^{2}} \frac{\pi^{2}}{16}\left\langle\Delta_{ \pm}^{2}\right\rangle, \quad a_{+-}=\frac{1}{N_{+} N_{-}} \frac{\pi^{2}}{16}\left\langle\Delta_{+} \Delta_{-}\right\rangle
$$

Similarly we find

$$
b_{++}=b_{--}=\frac{\pi^{2}}{16}\left\langle\Delta_{ \pm}^{2}\right\rangle, \quad b_{+-}=\frac{\pi^{2}}{16}\left\langle\Delta_{+} \Delta_{-}\right\rangle .
$$




\section{Discussion}

One should be aware that the observables defined in the previous section are $\mathcal{P}$ and $\mathcal{T}$ even. For vanishing net charge density per unit rapidity at $\eta=0$, the invariance under $\mathcal{C}$ predicts that $a_{++}=a_{--}$and $b_{++}=b_{--}$. Hence not only the Chiral Magnetic Effect contributes to the observables, but also some $\mathcal{P}$ even processes could do in principle. In order to find out whether the signal seen in Ref. [50] is really due to the Chiral Magnetic Effect one has to perform a detailed analysis and see whether calculations based on the Chiral Magnetic Effect are consistent with the data, and rule out more mundane explanations. Based on our main results Eqs. (40) and (41) we therefore will make some predictions. Let us make a cautionary statement here. We have made many approximations and there are many uncertainties. We expect that at best we might describe the data only semi-quantitatively.

\subsection{Absolute Value of Asymmetry}

In Eq. (43) we estimated with large uncertainty $\left\langle\Delta_{ \pm}^{2}\right\rangle=0.1$ at $b / R=1.6$ which corresponds roughly to $50-60 \%$ centrality. Using that that there are about 24 charged particles produced within $|\eta|<0.5$ (see Table 1) we find $a_{++}=a_{--} \sim 10^{-4}$. Now this estimate is very uncertain, we can easily increase or decrease it by an order of magnitude. However it seems comparing this number to the results presented in Ref. [50] that our prediction has about the same order of magnitude as seen in experiment. The main lesson from this is that although we are very uncertain about the value of the asymmetry, the Chiral Magnetic Effect does not give rise to an asymmetry which is many orders of magnitude away from the data obtained by experiment.

\subsection{Z Dependence}

The $Z$ (charge/atomic number) is probably our most robust prediction. Clearly the magnetic field is proportional to $Z$. Since the polarization of the quarks is proportional to $B$, the correlators $b_{a b}$ are proportional to $Z^{2}$. If polarization would become maximal however, which we believe is unlikely, there will be no $Z$-dependence. In principle one could test $Z$-dependence by taking nuclei with the same $A$ and different $Z$. This however could be difficult since it will require very accurate data.

\subsection{A Dependence}

The A dependence of our result depends upon which time scale is most important, that associated with the Lorentz contracted size of the nuclei, or that of the inverse saturation momenta. The specific $A$ dependence depends upon what range of impact parameter, beam energy and nuclear size we consider. 


\subsection{Beam Energy Dependence}

We are very uncertain about the beam energy dependence, since our result will depend strongly on what we take as our initial time. If the initial time scales with the longitudinal size of the nucleus, $R \exp \left(-Y_{0}\right)$, we expect no beamenergy dependence at all for $b_{a b}$. However if it turns out to be better to use the inverse saturation momentum for initial time, we can get a large dependence. In such case we always expect (as long as the pancake approximation stays good and a quark gluon plasma is formed) that $a_{a b}$ and $b_{a b}$ are smaller at larger beam energies, we never expect them to become larger.

\subsection{Centrality Dependence}

The centrality dependence of the correlators $a_{a b}$ and $b_{a b}$ has a strong dependence on initial time and could also have beam energy dependence. Let us assume that $\tau_{i}=\zeta R \exp \left(-Y_{0}\right)$, we can then use Eq. (40) to determine the centrality dependence of $a_{++}, a_{--}$and $b_{--}, b_{++}$. For now we will take $\zeta=2$ and $\lambda=1 \mathrm{fm}$ and present a plot of $a_{a b}$ of our model for Gold-Gold. It could be very well argued that one should take other values, but these seem to us not so unreasonable. In order to compare our results with the data we will plot $a_{++}$, $a_{--}$as a function of centrality and normalize the value in the 50-60 \% centrality bin to $7 \times 10^{-4}$ which is the value found by experiment [50]. To convert the impact parameter to centrality we used a table provided in Ref. [61]. Using their results we also computed the number of charged particles produced in each centrality bin (see also Ref. 62] for experimental data). These results are summarized in Table 1. Our result for $a_{++}$and $a_{--}$is displayed in Fig. 3. Qualitatively the result agrees with the data presented in Ref. [50].

\subsection{Suppression of $a_{+-}$Relative to $a_{++}$and $a_{--}$}

In Sec. [5 we found that $\left|\left\langle\Delta_{+} \Delta_{-}\right\rangle\right| \leq\left\langle\Delta_{ \pm}^{2}\right\rangle$ because of the screening effect. As a result $\left|a_{+-}\right| \leq a_{++}$. So it is very natural that the absolute value of $a_{+-}$is smaller than that of $a_{++}$. It easily follows from our results that

$$
\frac{\left|a_{+-}\right|}{a_{++}}=\frac{h(b / R, \lambda / R)}{g(b / R, \lambda / R)},
$$

where the functions $g(b / R, \lambda / R)$ and $h(b / R, \lambda / R)$ are given in Eq. (35)). We displayed $\left|a_{+-}\right| / a_{++}$as a function of $b / R$ in Fig. (4) for different values of $\lambda / R$. To compute the functions $g(b / R, \lambda / R)$ and $h(b / R, \lambda / R)$ we used the screening suppression factor $\xi_{ \pm}\left(x_{\perp}\right)$ given in Eq. (27). It could very well be that this simple suppression is to simplistic, and has to be modified somehow. Especially near the surface, and at large impact parameter, the function could be different. Nevertheless, one can infer from Fig. (4) that for reasonable values of $\lambda / R$ one finds an order of magnitude suppression of $a_{+-}$compared to $a_{++}$for small impact parameters which is consistent with data presented in 


\begin{tabular}{c|ccc} 
centrality & $b / R$ & $N_{\text {part }}$ & $\left(N_{+}+N_{-}\right) / 2$ \\
\hline $0-5 \%$ & 0.35 & 344 & 283 \\
$5-10 \%$ & 0.47 & 317 & 256 \\
$10-20 \%$ & 0.82 & 222 & 169 \\
$20-30 \%$ & 1.07 & 153 & 111 \\
$30-40 \%$ & 1.26 & 102 & 72 \\
$40-50 \%$ & 1.43 & 64 & 43 \\
$50-60 \%$ & 1.58 & 38 & 24 \\
$60-70 \%$ & 1.72 & 20 & 12 \\
$70-80 \%$ & 1.84 & 9.5 & 5.5
\end{tabular}

Table 1

Relation between centrality, impact parameter, number of participants $N_{\text {part }}$, and the average number of produced charged particles $\left(N_{+}+N_{-}\right) / 2$ within $|\eta|<0.5$ for Gold-Gold collisions at $130 \mathrm{GeV}$ per nucleon pair.

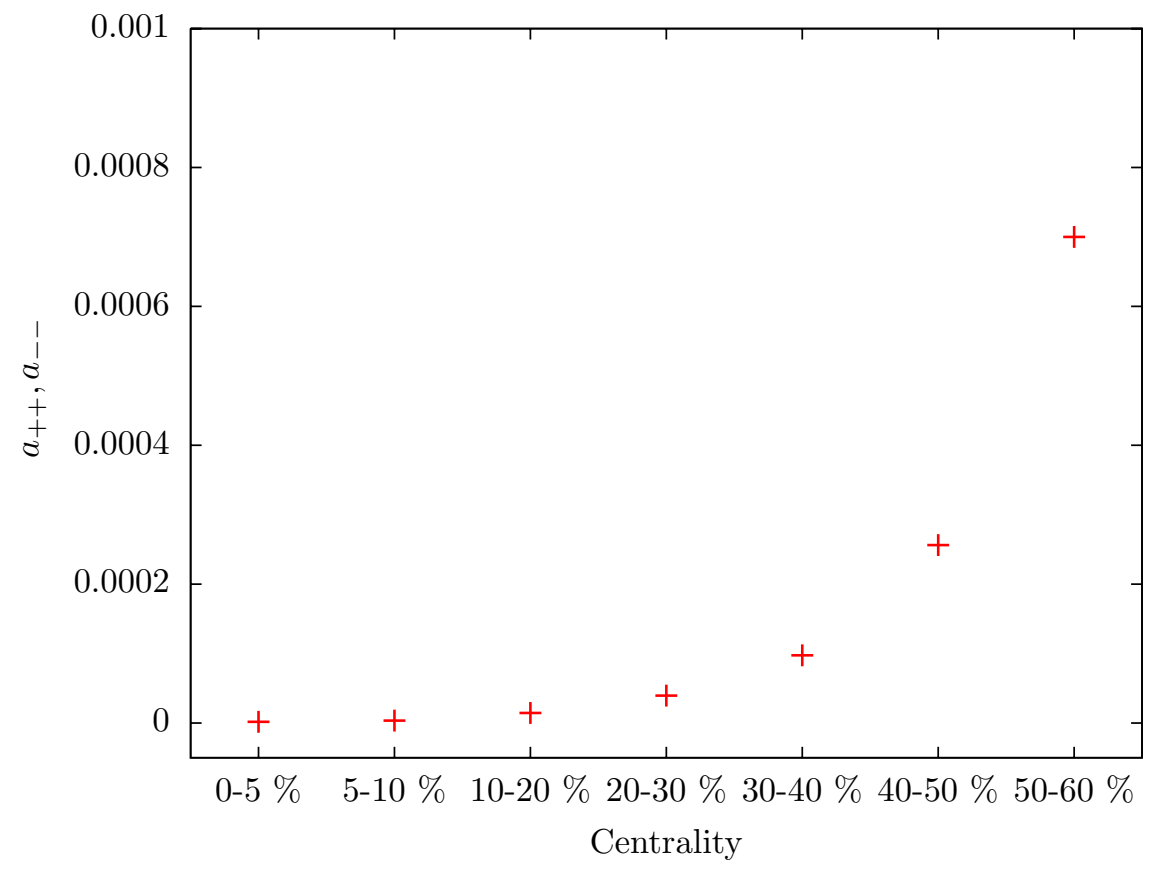

Fig. 3. Possible result of the Chiral Magnetic Effect for $a_{++}$and $a_{--}$as a function of centrality, for Gold-Gold collisions at $130 \mathrm{GeV}$ per nucleon pair.

Ref. [50]. For larger impact parameters the suppression is smaller, since the system size is in that case smaller.

Since the screening length $\lambda$ is independent of the size of the system, we expect $\left|a_{+-}\right| / a_{++}$and $\left|b_{+-}\right| / b_{++}$to be larger for smaller systems. This implies 


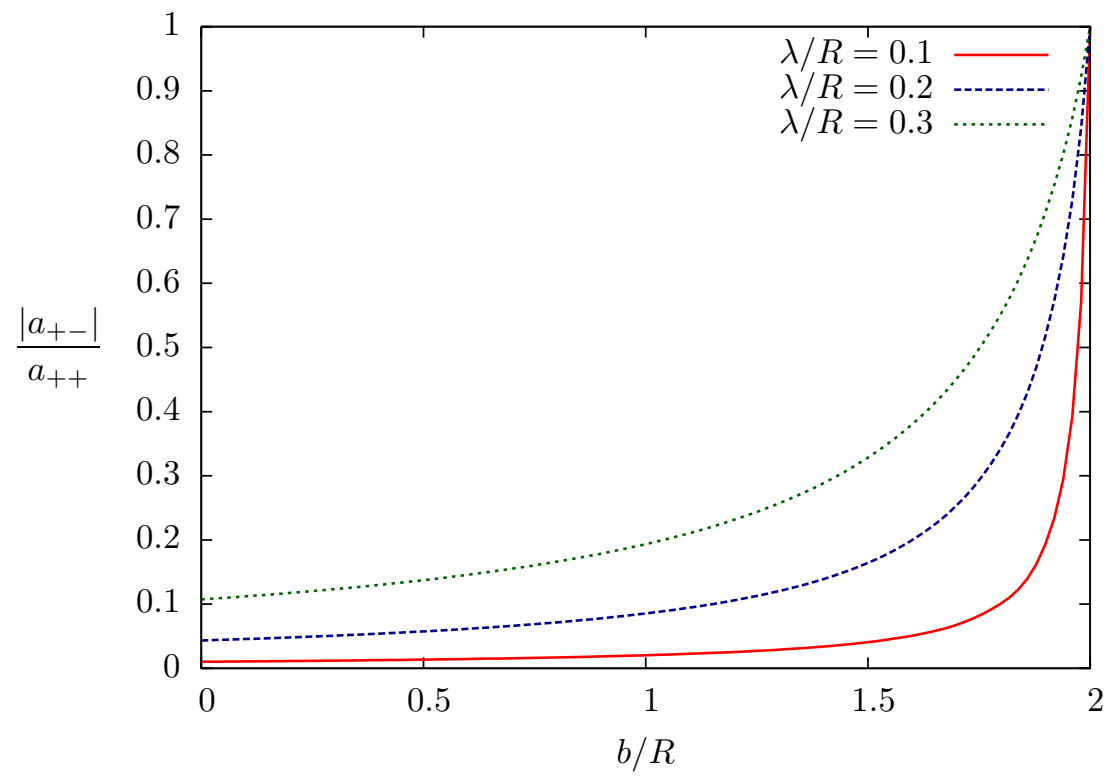

Fig. 4. Possible result of the correlator $\left|a_{+-}\right| / a_{++}$as a function of $b / R$ for different values of the screening length $\lambda / R$.

that $\left|a_{+-}\right| / a_{++}$and $\left|b_{+-}\right| / b_{++}$should be larger in Copper-Copper collisions than in Gold-Gold at the same centrality and energy.

\subsection{Transverse Momentum Dependence}

We expect that the particles which make the helicity flip have momentum in the order of $\alpha T$, hence we expect that the main contribution to our $b_{a b}$ comes from particles which have low transverse momentum. However we cannot clearly determine what is low. To make a safe prediction we expect the main contribution to $b_{a b}$ come from particles which have transverse momentum smaller than $1 \mathrm{GeV}$.

\subsection{Particle Species Dependence}

We think the main contribution to $b_{a b}$ should come from pions, since the Chiral Magnetic Effect also creates an isospin difference, but no baryon number difference if $N_{f}=2$ (see also Appendix B). However at this point we cannot make definite statements about the ratios protons to pions or kaons to pions that contributes to $b_{a b}$.

\subsection{Order Parameter for Chiral Symmetry/Confinement?}

Since it is absolutely necessary for the Chiral Magnetic Effect to operate that chiral symmetry is restored and matter is in the deconfined phase, measurement of $a_{a b} / b_{a b}$ if due to the chiral magnetic effect could also serve as an order parameter for chiral symmetry and confinement. If the temperature (beam energy) is too low such that there is chirally broken confined matter created 
we think it is likely that $a_{a b}$ and $b_{a b}$ should vanish or at least become much smaller.

\section{Summary}

The Chiral Magnetic Effect allows one to in principle probe the effects of topological charge changing processes in QCD. These phenomena are of great interest in field theory, and of great potential application in cosmology. In QCD, such phenomena are perhaps at the generation of nucleon mass, and also are associated with dynamical breaking of $\mathcal{P}$ and $\mathcal{C P}$ symmetries.

A confirmed observation of such an effect would be of great import. A confirmed observation must also be subject to alternative hypothesis. Our explanation has the advantage that many of its features may be varied by choice of beam energy, and the $\mathrm{Z}$ and $\mathrm{A}$ of the colliding nuclei. The computations we present are at present semi-quantitative estimates, that can be refined by more careful treatment of the magnetic field, and a better computation of the underlying topological charge changing processes.

\section{Acknowledgments}

We would like to thank Vasily Dzordzhadze, Jianwei Qiu, Ilya Selyuzhenkov, Yannis Semertzidis and Sergei Voloshin for discussions. At various stages of this work we have benefited from communications with Robert Pisarski, Mikhail Shaposhnikov, Edward Shuryak and Ariel Zhitnitsky. This manuscript has been authored under Contract No. \#DE-AC02-98CH10886 with the U.S. Department of Energy.

\section{A Magnetic Field in Heavy Ion Collisions}

Let us estimate the size of the magnetic field achieved in a heavy ion collision. We consider two similar nuclei with charge $Z$ and radius $R$. These nuclei are traveling with rapidity $Y_{0}$ in the positive and negative $z$-direction. At $t=0$ they collide with impact parameter $b$. We take the center of the nuclei at $t=0$ at $x= \pm b / 2$ so that the impact parameter direction lies along the $x$-axis. We depicted this situation in Fig. 2 ,

The nuclei in typical heavy-ion collision experiments are nearly traveling with the speed of light. For example gold-gold collisions at RHIC are performed at center of mass energies of $200 \mathrm{GeV}$ per nucleon pair which corresponds to $Y_{0} \approx 5$.4. In that case the Lorentz contraction factor $\gamma$ is equal to 100. Hence the nuclei are Lorentz contracted in the $z$-direction to 1 percent of their original size. Therefore to good approximation we can consider the two including nuclei as pancake shaped. As a result we can assume that number 
densities of the two nuclei are given by

$$
\rho_{ \pm}\left(\boldsymbol{x}_{\perp}^{\prime}\right)=\frac{2}{\frac{4}{3} \pi R^{3}} \sqrt{R^{2}-\left(\boldsymbol{x}_{\perp}^{\prime} \pm \boldsymbol{b} / 2\right)^{2}} \theta_{ \pm}\left(\boldsymbol{x}_{\perp}^{\prime}\right)
$$

here $\boldsymbol{x}_{\perp}^{\prime}$ denotes a vector transverse to the beam axis $\boldsymbol{e}_{z}$ and

$$
\theta_{ \pm}\left(\boldsymbol{x}_{\perp}^{\prime}\right)=\theta\left[R^{2}-\left(\boldsymbol{x}_{\perp}^{\prime} \pm \boldsymbol{b} / 2\right)^{2}\right]
$$

The functions $\theta_{ \pm}\left(\boldsymbol{x}_{\perp}^{\prime}\right)$ are the projections of the nuclei on the plane transverse to the beam axis and will also be used to separate the spectators from the participants. The number densities are normalized such that

$$
\int \mathrm{d}^{2} \boldsymbol{x}_{\perp}^{\prime} \rho_{ \pm}\left(\boldsymbol{x}_{\perp}^{\prime}\right)=1 .
$$

First let us quote the magnetic field at position $\boldsymbol{x}=\left(\boldsymbol{x}_{\perp}, z\right)$ caused by a particle with charge $Z$ moving in the positive $z$-direction with rapidity $Y$. At $t=0$ the particle can be found at position $\boldsymbol{x}_{\perp}^{\prime}$. This magnetic field can be obtained by either boosting the electric field of a charge $Z$ or using the Liénard-Wiechert potentials. One finds $(e=|e|)$

$$
e \boldsymbol{B}(\boldsymbol{x})=Z \alpha_{E M} \sinh (Y) \frac{\left(\boldsymbol{x}_{\perp}^{\prime}-\boldsymbol{x}_{\perp}\right) \times \boldsymbol{e}_{z}}{\left[\left(\boldsymbol{x}_{\perp}^{\prime}-\boldsymbol{x}_{\perp}\right)^{2}+(t \sinh Y-z \cosh Y)^{2}\right]^{3 / 2}},
$$

Now we can estimate the strength of the magnetic field during a heavy ion collision. We are only interested in $t>0$, i.e. just after the collision. Then we can split the magnetic field in the following way

$$
\boldsymbol{B}=\boldsymbol{B}_{s}^{+}+\boldsymbol{B}_{s}^{-}+\boldsymbol{B}_{p}^{+}+\boldsymbol{B}_{p}^{-},
$$

where $\boldsymbol{B}_{s}^{ \pm}$and $\boldsymbol{B}_{p}^{ \pm}$are the contributions of the spectators and the participants moving in the positive or negative $z$-direction respectively. We assume that the spectators do not scatter at all and stay traveling with the beam rapidity $Y_{0}$. It follows that $\left(Y_{0}>0\right)$

$$
\begin{aligned}
e \boldsymbol{B}_{s}^{ \pm}\left(\tau, \eta, \boldsymbol{x}_{\perp}\right)= \pm Z \alpha_{E M} \sinh \left(Y_{0} \mp \eta\right) \int \mathrm{d}^{2} \boldsymbol{x}_{\perp}^{\prime} \rho_{ \pm}\left(\boldsymbol{x}_{\perp}^{\prime}\right)\left[1-\theta_{\mp}\left(\boldsymbol{x}_{\perp}^{\prime}\right)\right] \\
\times \frac{\left(\boldsymbol{x}_{\perp}^{\prime}-\boldsymbol{x}_{\perp}\right) \times \boldsymbol{e}_{z}}{\left[\left(\boldsymbol{x}_{\perp}^{\prime}-\boldsymbol{x}_{\perp}\right)^{2}+\tau^{2} \sinh \left(Y_{0} \mp \eta\right)^{2}\right]^{3 / 2}},
\end{aligned}
$$

where the proper time $\tau=\left(t^{2}-z^{2}\right)^{1 / 2}$ and the space-time rapidity $\eta=$ $\frac{1}{2} \log [(t+z) /(t-z)]$.

Many particles will be created by the interactions between the participants. However, the number produced positively and negatively charged particles will be nearly equal. Moreover the expansion of these produced particles is almost spherical. We therefore expect that the contribution of the produced particles 
to the magnetic field is very small and will neglect this contribution. Hence we only have to take into account the contribution of the participants which were originally there. It is know that these participants stay traveling along the beam axis according to the following normalized distribution

$$
f(Y)=\frac{a}{2 \sinh \left(a Y_{0}\right)} e^{a Y}, \quad-Y_{0} \leq Y \leq Y_{0}
$$

Experimental data shows that $a \approx 1 / 2$, consistent with the baryon junction stopping mechanism (see [63] and references therein).

The contribution of the participants to the magnetic field is hence given by

$$
\begin{array}{r}
e \boldsymbol{B}_{p}^{ \pm}\left(\tau, \eta, \boldsymbol{x}_{\perp}\right)= \pm Z \alpha_{E M} \int \mathrm{d}^{2} \boldsymbol{x}_{\perp}^{\prime} \\
\int_{-Y_{0}}^{Y_{0}} \mathrm{~d} Y f(Y) \sinh (Y \mp \eta) \rho_{ \pm}\left(\boldsymbol{x}_{\perp}^{\prime}\right) \theta_{\mp}\left(\boldsymbol{x}_{\perp}^{\prime}\right) \times \\
\frac{\left(\boldsymbol{x}_{\perp}^{\prime}-\boldsymbol{x}_{\perp}\right) \times \boldsymbol{e}_{z}}{\left[\left(\boldsymbol{x}_{\perp}^{\prime}-\boldsymbol{x}_{\perp}\right)^{2}+\tau^{2} \sinh (Y \mp \eta)^{2}\right]^{3 / 2}} . \quad(\mathrm{A} .8)
\end{array}
$$

We have evaluated the magnetic field numerically at the origin $(\eta=$ $\left.0, \boldsymbol{x}_{\perp}=0\right)$ in which case it is pointing in the $y$-direction. We took colliding gold ions $(Z=79, R=7 \mathrm{fm})$ with different beam rapidities $(Y=4.19$ and $Y=5.36$ ). The results are displayed in Figs. A.1 and A.2. Clearly enormous magnetic fields are created in non-central heavy ion collisions.

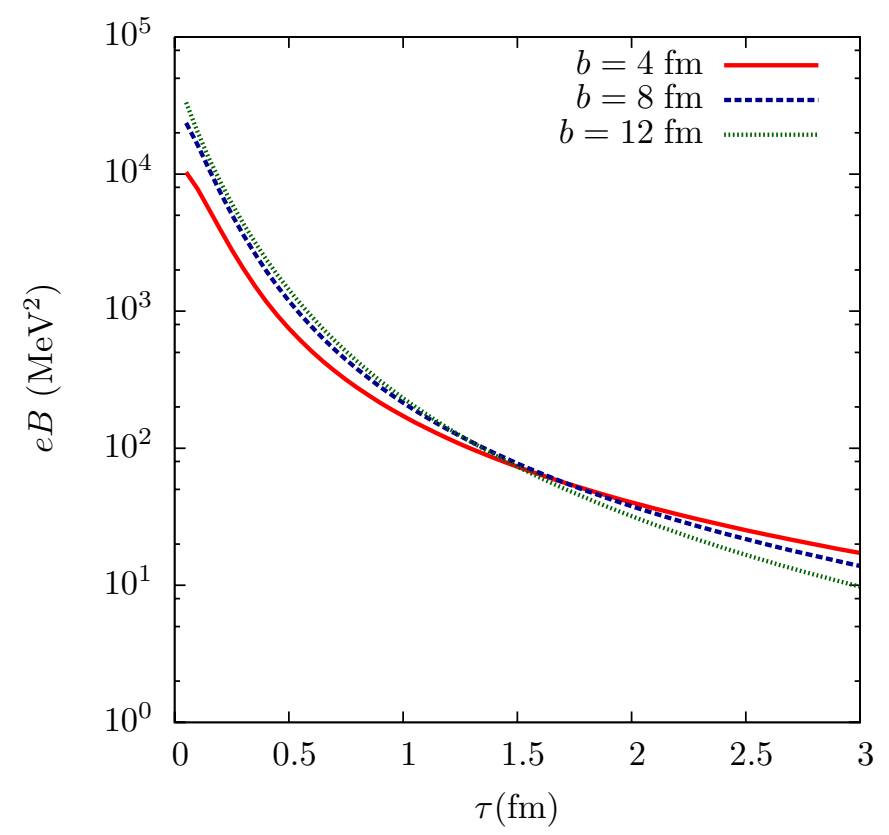

Fig. A.1. Magnetic field at the center of a gold-gold collision, for different impact parameters. Here the center of mass energy is $62 \mathrm{GeV}$ per nucleon pair $\left(Y_{0}=4.2\right)$.

For our purposes it is useful to have some analytic expression of the magnetic field. We can find reasonable approximations for $\tau \gtrsim 2 R / \sinh \left(Y_{0}\right)$. First 


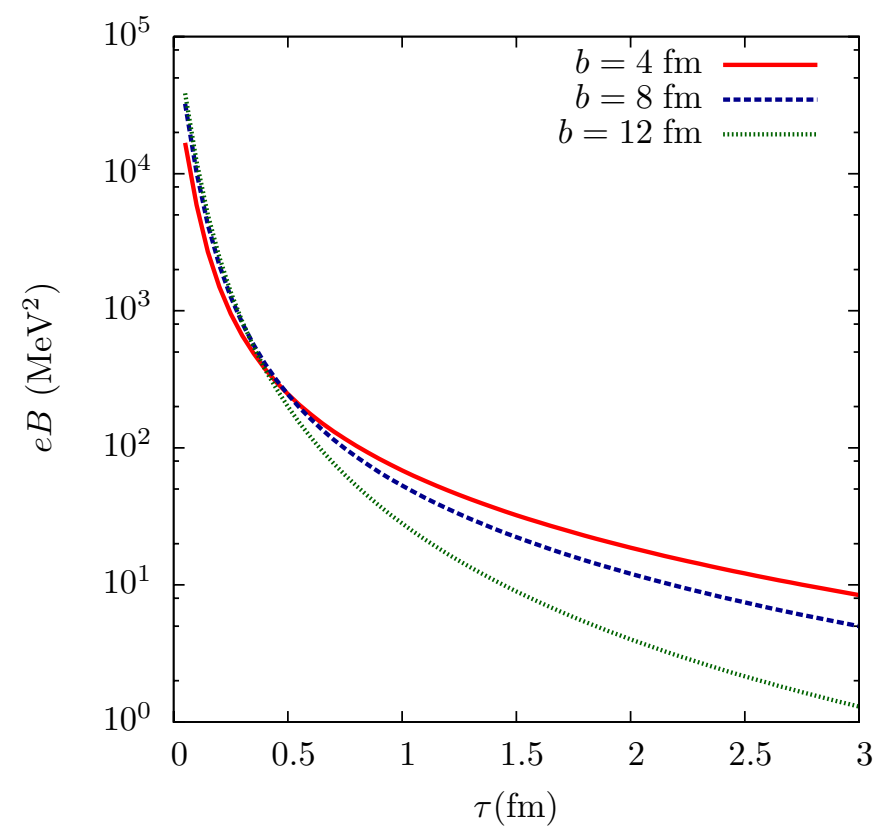

Fig. A.2. Magnetic field at the center of a gold-gold collision, for different impact parameters. Here the center of mass energy is $200 \mathrm{GeV}$ per nucleon pair $\left(Y_{0}=5.4\right)$.

we will consider the spectators, then we will discuss an approximation for the participants. We will perform both approximations at the origin $\left(\boldsymbol{x}_{\perp}=0\right.$ and $\eta=0$ ). In that case the magnetic field is pointing in the $y$-direction, $e \boldsymbol{B}=e B \boldsymbol{e}_{y}$. Especially for large impact parameters the magnetic field at the origin will be a good estimate for the magnetic field at the surface of the interacting region, since the magnetic field in the overlap region is to a good degree homogeneous in the transverse plane.

\section{A.1 Spectator Contribution for $\tau \gtrsim R / \sinh \left(Y_{0}\right)$}

For $\tau \gtrsim R \sinh \left(Y_{0}\right)$ the denominator of the integrand of the spectator contribution Eq. (A.6) can be approximated by $\tau^{3} \sinh \left(Y_{0}\right)^{3}$. Hence we find

$$
e B_{s} \approx Z \alpha_{E M} \exp \left(-2 Y_{0}\right) \frac{4 R}{\tau^{3}} g(b / R),
$$

where

$$
g(b / R)=\sum_{ \pm} g_{ \pm}(b / R)
$$

with

$$
g_{ \pm}(b / R)=\mp \frac{1}{R} \int \mathrm{d}^{2} x_{\perp}^{\prime} \rho_{ \pm}\left(\boldsymbol{x}_{\perp}^{\prime}\right)\left(1-\theta_{\mp}\left(\boldsymbol{x}_{\perp}^{\prime}\right)\right) x^{\prime} .
$$

We find that to very good approximation $g_{ \pm}(b / R)=b / R$. As a result

$$
e B_{s} \approx Z \alpha_{E M} \exp \left(-2 Y_{0}\right) \frac{4 b}{\tau^{3}}
$$




\section{A.2 Participant Contribution for $R / \sinh \left(Y_{0}\right) \lesssim \tau \lesssim R$}

At midrapidity $(\eta=0)$ the participant contribution to the magnetic field Eq. (A.8) contains the following integral over the beam rapidities

$$
I=\frac{a}{2 \sinh \left(a Y_{0}\right)} \frac{1}{\tau^{3}} \int_{-Y_{0}}^{Y_{0}} \mathrm{~d} Y \frac{\exp (a Y) \sinh (Y)}{\left[\beta^{2}+\sinh ^{2}(Y)\right]^{3 / 2}},
$$

where $\beta^{2}=\left(\boldsymbol{x}_{\perp}^{\prime}-\boldsymbol{x}_{\perp}\right)^{2} / \tau^{2}$. We are interested in finding an approximation to Eq. (A.13) for $1<\beta<\sinh \left(Y_{0}\right)$. The first step is to write

$$
I \approx \frac{a}{\exp \left(a Y_{0}\right)} \frac{1}{2 \tau^{3}} \int_{0}^{Y_{0}} \mathrm{~d} Y \frac{\exp [(1+a) Y]}{\left[\beta^{2}+\frac{1}{4} \exp (2 Y)\right]^{3 / 2}} .
$$

The last integral can be computed exactly. However, since it is quite complicated for $a=1 / 2$ and because we are anyway interested in the limit where $1<\beta<\sinh \left(Y_{0}\right)$ we will expand the result in $\beta / \sinh \left(Y_{0}\right)$. This gives for $a<1$,

where

$$
I \approx c \exp \left(-a Y_{0}\right) \frac{1}{\tau^{1+a}} \frac{1}{\left|\boldsymbol{x}_{\perp}^{\prime}-\boldsymbol{x}_{\perp}\right|^{2-a}},
$$

$$
c=\frac{2^{1+a} a \Gamma(1-a / 2) \Gamma(3 / 2+a / 2)}{(1+a) \sqrt{\pi}} .
$$

For $a=1 / 2$ we find $c=0.59907 \ldots$.

Now let us try to approximate the magnetic field at the origin $\left(\eta=0, \boldsymbol{x}_{\perp}=\right.$ 0 ) using the approximation for $I$. Taking $a=1 / 2$ we find that

$$
e B_{p} \approx c Z \alpha_{E M} \exp \left(-Y_{0} / 2\right) \frac{1}{R^{1 / 2} \tau^{3 / 2}} f(b / R)
$$

where

$$
f(b / R)=\sum_{ \pm} f_{ \pm}(b / R)
$$

with

$$
f_{ \pm}(b / R)=\mp R^{1 / 2} \int \mathrm{d}^{2} x_{\perp}^{\prime} \rho_{ \pm}\left(\boldsymbol{x}_{\perp}^{\prime}\right) \theta_{\mp}\left(\boldsymbol{x}_{\perp}^{\prime}\right) \frac{x^{\prime}}{\left|\boldsymbol{x}_{\perp}^{\prime}\right|^{3 / 2}} .
$$

We have computed $f(b / R)$ numerically and displayed the result in Fig. (A.2).

\section{B Alternative derivation of Chiral Magnetic Effect}

We will show in a somewhat more formal way than in Sec. 4 that a homogeneous background magnetic field in the presence of a gauge field configuration with nonzero $Q_{\mathrm{w}}$ gives rise to a vector current $j_{\mu}=\left\langle\bar{\psi} \gamma_{\mu} \psi\right\rangle_{A}$ along the direction of the magnetic field.

We take the background magnetic field to point in the $z$-direction so that only the following component is non-vanishing,

$$
j_{3}=\left\langle\psi \gamma_{3} \psi\right\rangle_{A}=\left\langle\psi_{R}^{\dagger} \sigma_{3} \psi_{R}\right\rangle_{A}-\left\langle\psi_{L}^{\dagger} \sigma_{3} \psi_{L}\right\rangle_{A}
$$




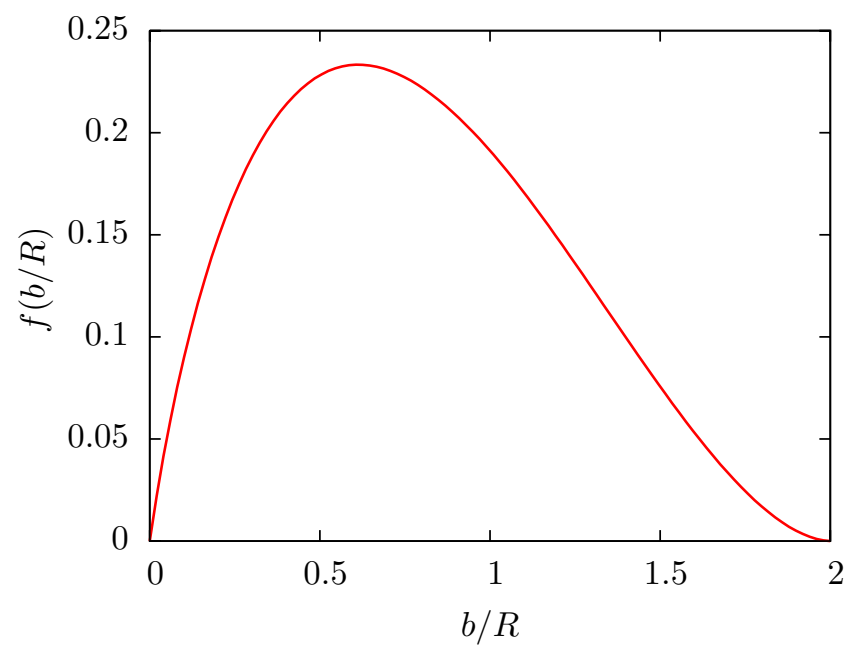

Fig. A.3. Plot of the function $f(b / R)$. This function describes the impact parameter dependence of the approximated participant contribution to the magnetic field and is defined in Eq. (A.18).

Initially, there is no difference between the number of left-handed and righthanded handed modes, therefore $j_{3}$ vanishes and there is no charge separation. Clearly Eq. (B.1) shows that if left- and right-handed modes are polarized in the same direction (as it should in a large magnetic field), a charged current will only form if there is a difference between $N_{R}$ and $N_{L}$. More explicitly in the limit of a very large magnetic field $\left(e B \gg T^{2}\right.$ or $e B \gg p_{F}^{2}$, where $p_{F}$ is the Fermi momentum)

$$
\int \mathrm{d}^{3} x\left\langle\psi_{R, L}^{\dagger} \sigma_{3} \psi_{R, L}\right\rangle_{A}=\operatorname{sgn}(q) \int \mathrm{d}^{3} x\left\langle\psi_{R, L}^{\dagger} \psi_{R, L}\right\rangle_{A}
$$

Here $q$ denotes the charge of the particle and reflects the fact that particles with different charge are polarized oppositely in a magnetic field. We now immediately find that in the limit of large magnetic field the total current for a flavor $f$ is given by

$$
J_{3}^{f}=\int \mathrm{d}^{3} x j_{3}^{f}(x)=\operatorname{sgn}\left(q_{f}\right)\left(N_{R}^{f}-N_{L}^{f}\right)
$$

In the chiral limit this implies that at $t=\infty$ an electromagnetic (EM) and a baryon (B) current is formed which magnitude equals

$$
J_{3}^{E M}=2 Q_{\mathrm{w}} \sum_{f}\left|q_{f}\right|, \quad J_{3}^{B}=\frac{2}{3} Q_{\mathrm{w}} \sum_{f} \operatorname{sgn}\left(q_{f}\right) .
$$

If we assume that all quarks were located within a finite volume, this electromagnetic current generates a charge difference $Q$ between two sides of 
a plane perpendicular to the magnetic field of size

$$
Q=2 Q_{\mathrm{w}} \sum_{f}\left|q_{f}\right|
$$

This difference is the same as we found before in Eq. (16).

In the same way we find the following baryon number difference

$$
B=2 \sum_{f} J_{3}^{f}=\frac{2}{3} Q_{w} \sum_{f} \operatorname{sgn}\left(q_{f}\right) .
$$

The baryon number difference vanishes for $N_{f}=2$, while for $N_{f}=3, B=$

$\frac{2}{3} Q_{\mathrm{w}}$. Since $B$ times the average charge of a baryon is smaller than $Q$, we expect the charge difference mainly to be realized in mesons, like $\pi^{+}$and $\pi^{-}$.

In this appendix we considered a gauge field configuration with nonzero $Q_{\mathrm{w}}$ in the presence of a very large homogeneous magnetic field. It would be interesting to extend these results by studying the behavior of the vector current in a smaller magnetic field.

\section{References}

[1] A. A. Belavin, A. M. Polyakov, A. S. Shvarts and Yu. S. Tyupkin, Phys. Lett. B 59, 85 (1975).

[2] G. 't Hooft, Phys. Rev. Lett. 37, 8 (1976); G. 't Hooft, Phys. Rev. D 14, 3432 (1976) [Erratum-ibid. D 18, 2199 (1978)].

[3] R. Jackiw and C. Rebbi Phys. Rev. Lett. 37, 172 (1976).

[4] S. S. Chern and J. Simons, Annals Math. 99, 48 (1974)

[5] S. L. Adler, Phys. Rev. 177, 2246 (1969).

[6] J. S. Bell and and R. Jackiw, Nuovo Cim. A60, 47 (1969).

[7] N. S. Manton Phys. Rev. D28, 2019 (1983).

[8] F. R. Klinkhamer and N. S. Manton, Phys. Rev. D 30, 2212 (1984).

[9] V. A. Kuzmin, V. A. Rubakov and M. E. Shaposhnikov, Phys. Lett. B 155, 36 (1985).

[10] M. E. Shaposhnikov, Nucl. Phys. B 287, 757 (1987).

[11] P. Arnold and L. D. McLerran, Phys. Rev. D 36, 581 (1987).

[12] P. Arnold and L. D. McLerran, Phys. Rev. D 37, 1020 (1988).

[13] L. D. McLerran, E. Mottola and M. E. Shaposhnikov, Phys. Rev. D 43, 2027 (1991). 
[14] R. D. Peccei and H. R. Quinn, Phys. Rev. D 16, 1791 (1977); S. Weinberg, Phys. Rev. Lett. 40, 223 (1978); F. Wilczek, Phys. Rev. Lett. 40, 279 (1978).

[15] J.E. Kim, Phys. Rev. Lett. 43 (1979) 103; M.A. Shifman, A.I. Vainshtein, and V.I. Zakharov, Nucl. Phys. B166, 493 (1980).

[16] M. Dine, W. Fischler, and M. Srednicki, Phys. Lett. B104 (1981) 199; A.R. Zhitnitsky, Yad.Fiz. 31 (1980) 497; Sov. J. Nucl. Phys. 31 (1980) 260 (DFSZaxion).

[17] M. Srednicki, hep-th/0210172; R. D. Peccei, arXiv:hep-ph/0607268; P. Sikivie, arXiv:astro-ph/0610440.

[18] T. D. Lee and G. C. Wick, Phys. Rev. D 9, 2291 (1974).

[19] T. D. Lee, Phys. Rev. D 8, 1226 (1973).

[20] P. D. Morley and I. A. Schmidt, Z. Phys. C 26, 627 (1985).

[21] A. A. Anselm, Phys. Lett. B 217, 169 (1989); A. A. Anselm and M. G. Ryskin, Phys. Lett. B 266, 482 (1991); J. P. Blaizot and A. Krzywicki, Phys. Rev. D 46, 246 (1992); J. D. Bjorken, K. L. Kowalski and C. C. Taylor, arXiv:hep-ph/9309235; K. Rajagopal and F. Wilczek, Nucl. Phys. B 404, 577 (1993) [arXiv:hep-ph/9303281].

[22] D. Kharzeev, R. D. Pisarski and M. H. G. Tytgat, Phys. Rev. Lett. 81, 512 (1998); arXiv:hep-ph/0012012.

[23] D. Kharzeev and R. D. Pisarski, Phys. Rev. D 61, 111901 (2000) arXiv:hep-ph/9906401.

[24] M. A. Shifman, Prog. Part. Nucl. Phys. 39, 1 (1997) arXiv:hep-th/9704114; I. I. Kogan, A. Kovner and M. A. Shifman, Phys. Rev. D 57, 5195 (1998) arXiv:hep-th/9712046.

[25] E. Witten, Phys. Rev. Lett. 81, 2862 (1998) arXiv:hep-th/9807109].

[26] I. E. Halperin and A. Zhitnitsky, Phys. Rev. D 58, 054016 (1998) arXiv:hep-ph/9711398]; Phys. Rev. Lett. 81, 4071 (1998) arXiv:hep-ph/9803301]; T. Fugleberg, I. E. Halperin and A. Zhitnitsky, Phys. Rev. D 59, 074023 (1999) arXiv:hep-ph/9808469]; R. H. Brandenberger, I. E. Halperin and A. Zhitnitsky, arXiv:hep-ph/9808471.

[27] C. Vafa and E. Witten, Phys. Rev. Lett. 53, 535 (1984); Nucl. Phys. B 234, 173 (1984).

[28] D. T. Son and M. A. Stephanov, Phys. Rev. Lett. 86, 592 (2001) arXiv:hep-ph/0005225]; Phys. Atom. Nucl. 64, 834 (2001) [Yad. Fiz. 64, 899 (2001)] [arXiv:hep-ph/0011365].

[29] T. D. Cohen, Phys. Rev. D 64, 047704 (2001) arXiv:hep-th/0101197.

[30] A. B. Migdal, Zh. Eksp. Teor. Fiz. 61 (1971) 2210 [Sov. Phys. JETP 36 (1973) 1052]; Rev. Mod. Phys. 50 (1978) 107. 
[31] A. A. Andrianov and D. Espriu, arXiv:0709.0049 [hep-ph].

[32] R. D. Pisarski and D. H. Rischke, Phys. Rev. Lett. 83, 37 (1999) arXiv:nucl-th/9811104.

[33] S. Aoki, Phys. Rev. D 30, 2653 (1984); S. Aoki and A. Gocksch, Phys. Rev. D 45, 3845 (1992).

[34] R. F. Dashen, Phys. Rev. D 3, 1879 (1971).

[35] M. Creutz, Phys. Rev. Lett. 92, 201601 (2004) arXiv:hep-lat/0312018]; arXiv:hep-ph/0312225.

[36] S. Bronoff and C. P. Korthals Altes, Phys. Lett. B 448, 85 (1999) arXiv:hep-ph/9811243.

[37] V. Azcoiti and A. Galante, Phys. Rev. Lett. 83, 1518 (1999) arXiv:hep-th/9901068; X. d. Ji, Phys. Lett. B 554, 33 (2003) arXiv:hep-ph/0108162]; M. B. Einhorn and J. Wudka, Phys. Rev. D 67, 045004 (2003) arXiv:hep-ph/0205346.

[38] K. Buckley, T. Fugleberg and A. Zhitnitsky, Phys. Rev. Lett. 84, 4814 (2000) arXiv:hep-ph/9910229]; D. Ahrensmeier, R. Baier and M. Dirks, Phys. Lett. B 484, 58 (2000) |arXiv:hep-ph/0005051|; E. V. Shuryak and A. R. Zhitnitsky, Phys. Rev. C 66, 034905 (2002) [arXiv:hep-ph/0111352]; A. K. Chaudhuri, Phys. Rev. C 65, 024906 (2002) arXiv:hep-ph/0109211].

[39] D. Kharzeev, A. Krasnitz and R. Venugopalan, Phys. Lett. B 545, 298 (2002) arXiv:hep-ph/0109253.

[40] T. Lappi and L. McLerran, Nucl. Phys. A 772, 200 (2006) arXiv:hep-ph/0602189]; T. Lappi, J. Phys. G 32, S179 (2006) arXiv:hep-ph/0606090; Phys. Lett. B 643, 11 (2006) arXiv:hep-ph/0606207.

[41] S. Moch, A. Ringwald and F. Schrempp, Nucl. Phys. B 507, 134 (1997) arXiv:hep-ph/9609445.

[42] D. E. Kharzeev, Y. V. Kovchegov and E. Levin, Nucl. Phys. A 690, 621 (2001) arXiv:hep-ph/0007182; Nucl. Phys. A 699, 745 (2002) arXiv:hep-ph/0106248.

[43] E. V. Shuryak and I. Zahed, Phys. Rev. D 62, 085014 (2000) arXiv:hep-ph/0005152.

[44] M. A. Nowak, E. V. Shuryak and I. Zahed, Phys. Rev. D 64, 034008 (2001) arXiv:hep-ph/0012232.

[45] R. A. Janik, E. Shuryak and I. Zahed, Phys. Rev. D 67, 014005 (2003) arXiv:hep-ph/0206005.

[46] S. A. Voloshin, Phys. Rev. C 62, 044901 (2000) arXiv:nucl-th/0004042.

[47] L. E. Finch, A. Chikanian, R. S. Longacre, J. Sandweiss and J. H. Thomas, Phys. Rev. C 65, 014908 (2002); arXiv:nucl-th/0109078. 
[48] D. Kharzeev, Phys. Lett. B 633, 260 (2006); arXiv:hep-ph/0406125.

[49] S. A. Voloshin, Phys. Rev. C 70, 057901 (2004) arXiv:hep-ph/0406311.

[50] I. V. Selyuzhenkov [STAR Collaboration], Rom. Rep. Phys. 58, 049 (2006) arXiv:nucl-ex/0510069.

[51] D. Kharzeev and A. Zhitnitsky, arXiv:0706.1026 [hep-ph].

[52] E. Witten, Phys. Lett. B 86, 283 (1979).

[53] C. W. Bernard, Phys. Rev. D 19, 3013 (1979).

[54] R. D. Pisarski and L. G. Yaffe, Phys. Lett. B 97, 110 (1980).

[55] D. J. Gross, R. D. Pisarski and L. G. Yaffe, Rev. Mod. Phys. 53, 43 (1981).

[56] G. D. Moore, C. r. Hu and B. Muller, Phys. Rev. D 58, 045001 (1998) arXiv:hep-ph/9710436.

[57] D. Bödeker, G. D. Moore and K. Rummukainen, Phys. Rev. D 61, 056003 (2000) arXiv:hep-ph/9907545.

[58] L. McLerran and R. Venugopalan Phys. Rev. D49, 3352 (1994), Phys. Rev. D49, 2233 (1994).

[59] E. Iancu, A. Leonidov, and L. McLerran, Nucl. Phys. A692, 583 (2001); E. Iancu, E. Ferreiro, A. Leonidov and L. McLerran, Nucl. Phys. A692, $583(2001)$.

[60] D. Kharzeev, A. Krasnitz and R. Venugopalan, Phys. Lett. B545, 298 (2002).

[61] D. Kharzeev and M. Nardi, Phys. Lett. B507 121 (2001);

D. Kharzeev and E. Levin, Phys. Lett. B 523, 79 (2001) arXiv:nucl-th/0108006.

[62] S. S. Adler et al. [PHENIX Collaboration], Phys. Rev. C 71, 034908 (2005) [Erratum-ibid. C 71, 049901 (2005)] arXiv:nucl-ex/0409015].

[63] D. Kharzeev, Phys. Lett. B 378, 238 (1996) arXiv:nucl-th/9602027. 